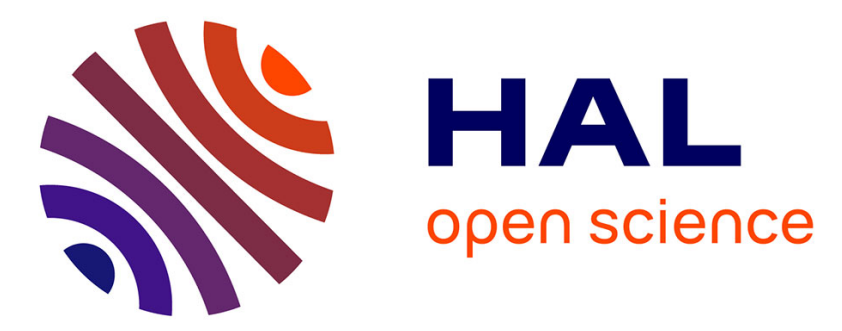

\title{
Bijective digitized rigid motions on subsets of the plane
} Kacper Pluta, Pascal Romon, Yukiko Kenmochi, Nicolas Passat

\section{To cite this version:}

Kacper Pluta, Pascal Romon, Yukiko Kenmochi, Nicolas Passat. Bijective digitized rigid motions on subsets of the plane. Journal of Mathematical Imaging and Vision, 2017, 59 (1), pp.84-105. 10.1007/s10851-017-0706-8 . hal-01497610

\section{HAL Id: hal-01497610 https://hal.science/hal-01497610}

Submitted on 28 Mar 2017

HAL is a multi-disciplinary open access archive for the deposit and dissemination of scientific research documents, whether they are published or not. The documents may come from teaching and research institutions in France or abroad, or from public or private research centers.
L'archive ouverte pluridisciplinaire HAL, est destinée au dépôt et à la diffusion de documents scientifiques de niveau recherche, publiés ou non, émanant des établissements d'enseignement et de recherche français ou étrangers, des laboratoires publics ou privés. 


\title{
Bijective digitized rigid motions on subsets of the plane
}

\author{
Kacper Pluta • Pascal Romon • Yukiko Kenmochi · Nicolas Passat
}

Received: date / Accepted: date

\begin{abstract}
Rigid motions in $\mathbb{R}^{2}$ are fundamental operations in 2D image processing. They satisfy many properties: in particular, they are isometric and therefore bijective. Digitized rigid motions, however, lose these two properties. To investigate the lack of injectivity or surjectivity and more generally their local behavior, we extend the framework initially proposed by Nouvel and Rémila to the case of digitized rigid motions. Yet, for practical applications, the relevant information is not global bijectivity, which is seldom achieved, but bijectivity of the motion restricted to a given finite subset of $\mathbb{Z}^{2}$. We propose two algorithms testing that condition. Finally, because rotation angles are rarely given with infinite precision, we propose a third algorithm providing optimal angle intervals that preserve this restricted bijectivity.
\end{abstract}

Kacper Pluta

Université Paris-Est, LIGM (UMR 8049), Marne-la-Vallée, France

Kacper Pluta and Pascal Romon

Université Paris-Est, LAMA (UMR 8050), UPEM, UPEC, CNRS, F-77454, Marne-la-Vallée, France

Yukiko Kenmochi

Université Paris-Est, LIGM (UMR 8049), UPEM, CNRS, ESIEE Paris, ENPC, Marne-la-Vallée, France

Nicolas Passat

Université de Reims Champagne-Ardenne, CReSTIC, France

\section{Introduction}

Rigid motions (i.e., rotations, translations and their compositions) defined on $\mathbb{Z}^{2}$ are simple yet crucial operations in many image processing applications involving 2D data: for example, in template matching [2] and object tracking [15]. One way to design rigid motions on $\mathbb{Z}^{2}$ is to combine continuous rigid motions defined on $\mathbb{R}^{2}$ with a digitization operator that maps the results back onto $\mathbb{Z}^{2}$. However, a digitized rigid motion, though uniformly "close" to its continuous analogue, often no longer satisfies the same properties. In particular, due to digitization, such transformations do not preserve distances. As a consequence, bijectivity and point connectivity are generally lost. In this context, it is useful to understand the combinatorial, geometrical and topological alterations associated with digitized rigid motions. More precisely, we aim to observe the impact of rigid motions on the structure of $\mathbb{Z}^{2}$ at a local scale. Few efforts were already devoted to such topic, in particular for digitized rotations. The most impacting works within this field are those proposed by Nouvel and Rémila [6], who developed a framework for studying local alterations of $\mathbb{Z}^{2}$ under digitized rotations, namely on certain patches of $\mathbb{Z}^{2}$. This combinatorial model of the local behavior led them to characterizing bijective digitized rotations [7], and more generally studying non-bijective ones [8]. 
In this context our contribution is threefold.

1. We first extend the aforementioned combinatorial model of the local behavior of rigid motions on $\mathbb{Z}^{2}[6,8]$ to (i) digitized rigid motions, and (ii) any neighborhood patches regardless of their size and shape. We call this local description a neighborhood motion maps. Focusing in particular on neighborhood motion maps of 4- and 8-neighborhoods, we characterize the bijective rigid motions on $\mathbb{Z}^{2}$, extending the characterization of bijective digitized rotations of [7].

2. We restrict then to the practical problem of verifying whether a prescribed subset of $\mathbb{Z}^{2}$ is transformed bijectively (or more precisely injectively) by a digitized rigid motion. To this end, the local approach of neighborhood motion maps is well suited and leads to an algorithmic answer. More precisely, two different algorithms are proposed, the performance of each depends on the ratio of the size of the subset to the complexity of the rigid motion, measured by the integers of the Pythagorean triples.

3. This algorithmic approach can be used for finding, for a given subset $S$ and an injective rigid motion on $S$, a range of nearby parameters ensuring injectivity, thereby offering a stability result. This is done by extending the concept of hinge angles $[2,9,14]$ to rigid motions.

This article is an extension of the conference paper [11]. Our new contributions compared to this preliminary work are, a study of neighborhood motion maps for the 4- and 8-neighborhoods together with their complete list, provided in Appendices $\mathrm{A}$ and $\mathrm{B}$ and computation of intervals of confidence for bijectivity preservation described in item 3 above.

The remainder of this article is organized as follows. In Section 2, we recall basic definitions on continuous and digitized rigid motions and neighborhood of an integer point. In Section 3, we generalize the study proposed by Nouvel and Rémila [6] to digitized rigid motions and any neighborhood. Sections 4-6 are devoted to a study of bijective, surjective and injective digitized rigid motions. In Section 7, we conclude this article and provide some perspectives. Finally, Appendices A and B provide a complete list of neighborhood motion maps for the 4- and 8-neighborhood, respectively.

\section{Basic notions}

\subsection{Rigid motions on $\mathbb{R}^{2}$}

Rigid motions on $\mathbb{R}^{2}$ are bijective isometric maps [3]; in particular, they preserve distances and angles. The set of rigid motions includes rotations (around the origin), translations, and their compositions. In $\mathbb{R}^{2}$ a rigid motion is then defined as a function

$$
\mid \begin{aligned}
\mathcal{U}: \mathbb{R}^{2} & \rightarrow \mathbb{R}^{2} \\
\mathbf{x} & \mapsto \mathbf{R x}+\mathbf{t}
\end{aligned}
$$

where $\mathbf{t}=\left(t_{x}, t_{y}\right) \in \mathbb{R}^{2}$ is a translation vector and

$\mathbf{R}=\left[\begin{array}{cc}\cos \theta & -\sin \theta \\ \sin \theta & \cos \theta\end{array}\right]$

is a rotation matrix with $\theta \in[0,2 \pi)$ its rotation angle. This leads to the representation of rigid motions by a triplet of parameters $\left(t_{x}, t_{y}, \theta\right) \in \mathbb{R}^{2} \times[0,2 \pi)$.

\subsection{Rigid motions on $\mathbb{Z}^{2}$}

According to Equation (1), we generally have $\mathcal{U}\left(\mathbb{Z}^{2}\right) \nsubseteq \mathbb{Z}^{2}$; in other words, a rigid motion applied to points with integer coordinates maps them onto points with real coordinates. As a consequence, in order to define digitized rigid motions as maps from $\mathbb{Z}^{2}$ to $\mathbb{Z}^{2}$, the most common solution is to apply rigid motions on $\mathbb{Z}^{2}$ as a part of $\mathbb{R}^{2}$, and then combine the real results with a digitization operator

$$
\mid \begin{aligned}
\mathcal{D}: \mathbb{R}^{2} & \rightarrow \mathbb{Z}^{2} \\
(x, y) & \mapsto\left(\left\lfloor x+\frac{1}{2}\right\rfloor,\left\lfloor y+\frac{1}{2}\right\rfloor\right)
\end{aligned}
$$

where $\lfloor z\rfloor$ denotes the largest integer not greater than $z$. Then, digitized rigid motions are defined by

$U=\mathcal{D} \circ \mathcal{U}_{\mid \mathbb{Z}^{2}}$.

Due to the behavior of $\mathcal{D}$ that maps $\mathbb{R}^{2}$ onto $\mathbb{Z}^{2}$, digitized rigid motions are, most of the time, nonbijective. In other words, while any point $\mathbf{y} \in \mathbb{R}^{2}$ is associated to a unique preimage point $\mathbf{x} \in \mathbb{R}^{2}$, such that $\mathcal{U}(\mathbf{x})=\mathbf{y}$, a point $\mathbf{y} \in \mathbb{Z}^{2}$ can be associated to several (resp. no) preimage point(s) $\mathbf{x} \in \mathbb{Z}^{2}$ for a 


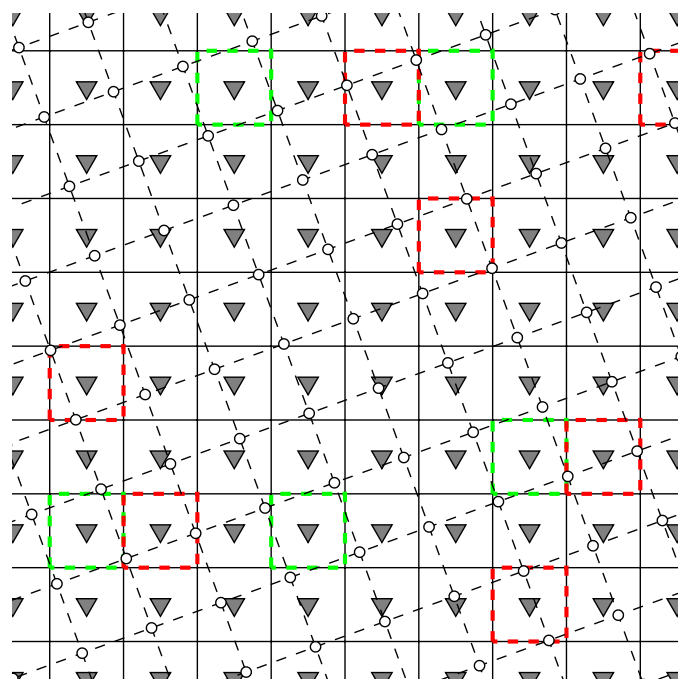

Fig. 1 Examples of three different point mappings: digitization cells corresponding to non-surjective, injective and non-injective mappings are in green, black and red, respectively. White dots indicate the positions of images of the points of the initial set $\mathbb{Z}^{2}$ by $\mathcal{U}$, embedded in $\mathbb{R}^{2}$, subdivided into digitization cells around the points of the final set $\mathbb{Z}^{2}$, represented by gray triangles.

digitized rigid motion $U$ associated to $\mathcal{U}$; in such case, $U$ is non-injective (resp. non-surjective). See Figure 1.

Remark 1 In $\mathbb{Z}^{2}$, a point can have either 0,1 or 2 preimages. In particular, when it has two preimages, $\mathbf{p}$ and $\mathbf{q}$, we have $|\mathbf{p}-\mathbf{q}|=1$ [5].

\subsection{Neighborhood motion map}

In $\mathbb{R}^{2}$, an intuitive way to define the neighborhood of a point $\mathbf{x}$ is to consider the set of points that lie within a ball of a given radius centered at $\mathbf{x}$. This metric definition actually remains valid in $\mathbb{Z}^{2}$, where it allows us to retrieve the classical notion of neighborhood based on adjacency relations.

Definition 1 (Neighborhood) The neighborhood of $\mathbf{p} \in \mathbb{Z}^{2}$ (of squared radius $r \in \mathbb{R}_{+}$), denoted $\mathcal{N}_{r}(\mathbf{p})$, is defined as

$$
\mathcal{N}_{r}(\mathbf{p})=\left\{\mathbf{p}+\mathbf{d} \in \mathbb{Z}^{2} \mid\|\mathbf{d}\|^{2} \leq r\right\} .
$$

In order to track these local alterations of the neighborhood of integer points, we introduce the notion of a neighborhood motion map, that is defined as a set of vectors; each representing information about a neighbor after rigid motion.

Definition 2 (Neighborhood motion map) Let $\mathbf{p} \in \mathbb{Z}^{2}$ and $r \in \mathbb{R}_{+}$. Let $U: \mathbb{Z}^{2} \rightarrow \mathbb{Z}^{2}$ be a digitized rigid motion. The neighborhood motion map of $\mathbf{p}$ with respect to $U$ and $r$ is the function defined as

$$
\mid \begin{aligned}
\mathcal{G}_{r}^{U}(\mathbf{p}): \mathcal{N}_{r}(\mathbf{0}) & \rightarrow \mathcal{N}_{r^{\prime}}(\mathbf{0}) \\
\mathbf{d} & \mapsto U(\mathbf{p}+\mathbf{d})-U(\mathbf{p})
\end{aligned}
$$

(with $r^{\prime} \geq r$ ). In other words, $\mathcal{G}_{r}^{U}(\mathbf{p})$ associates to each relative position of an integer point $\mathbf{q}=\mathbf{p}+\mathbf{d}$ in the neighborhood of $\mathbf{p}$, the relative position of the image $U(\mathbf{q})$ in the neighborhood of $U(\mathbf{p})$.

Note that a similar idea was previously proposed by Nouvel and Rémila [6] to track local alterations of the neighborhood $\mathcal{N}_{1}$ under $2 \mathrm{D}$ digitized rotations.

Remark 2 For the sake of readability, we will consider a visual representation of the $\mathcal{G}_{r}^{U}(\mathbf{p})$ functions as label maps. A first-reference-map $\mathcal{L}_{r}$ will associate a specific label to each point $\mathbf{q}-\mathbf{p}$ of $\mathcal{N}_{r}(\mathbf{0})$ for a given squared radius $\mathrm{r}$ (see Figure 2, for the maps $\mathcal{L}_{1}$ and $\mathcal{L}_{2}$ ). A second map $\mathcal{L}_{r}^{U}(\mathbf{p})-$ associated to $\mathcal{G}_{r}^{U}(\mathbf{p})$, i.e. to a point $\mathbf{p}$ and a digitized rigid motion $U$-will associate, to each point $\mathbf{r}$ of $\mathcal{N}_{r^{\prime}}(\mathbf{0})$, the labels of all the points $\mathbf{q}$ such that $U(\mathbf{q})-U(\mathbf{p})=\mathbf{r}$. Such a set of labels for each $\mathbf{r}$ may contain 0,1 or 2 labels, due to the possible mappings of integer points under digitized rigid motions (see examples in Figure 3).

\section{Remainder Range Partitioning and Neighborhood Motion Maps}

\subsection{Partitioning the remainder range}

Digitized rigid motions $U=\mathcal{D} \circ \mathcal{U}$ are piecewise constant, and thus non-continuous, which is a consequence of the nature of $\mathcal{D}$. In particular, for a given point $\mathbf{p} \in \mathbb{Z}^{2}$ and its neighborhood $\mathcal{N}_{r}(\mathbf{p})$, a slight modification of the parameters $\left(t_{x}, t_{y}, \theta\right)$ of $\mathcal{U}$ may result in a digitized motion $U$ such that $U(\mathbf{p})$ may move from one point of $\mathbb{Z}^{2}$ to another andmore importantly - such that the relative position 


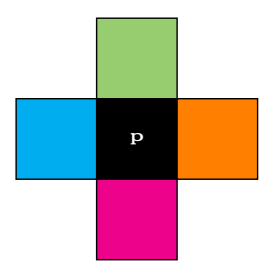

(a)

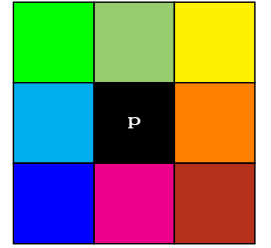

(b)
Fig. 2 The reference label maps $\mathcal{L}_{1}$ (a) and $\mathcal{L}_{2}$ (b).

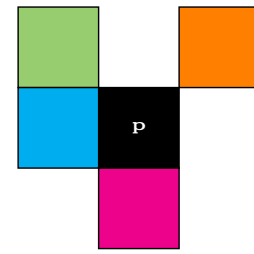

(a)

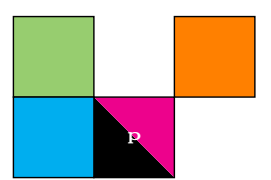

(b)
Fig. 3 Examples of label maps $\mathcal{L}_{1}^{U}(\mathbf{p})$. (a) Each point contains at most one label: the rigid motion $U$ is then locally injective. (b) One point contains two labels: $U$ is then noninjective.

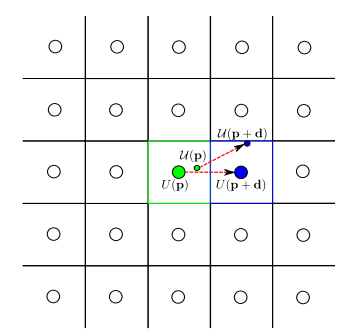

(a)

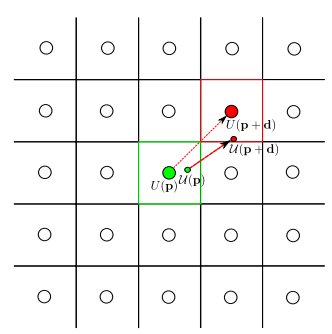

(b)
Fig. 4 An example of discontinuity of $U$, and thus of $\mathcal{G}_{r}^{U}(\mathbf{p})$. In (a) and (b), the image $\mathcal{U}(\mathbf{p})$ remains within the same unit square centered around the integer point depicted in green; thus the image $U(\mathbf{p})$ is the same for the two digitized rigid motions $U$ associated to the continuous rigid motions $\mathcal{U}$, that slightly differ with respect to the parameters $\left(t_{x}, t_{y}, \theta\right)$. However, the image of the point $\mathbf{p}+\mathbf{d}$, that belongs to the neighborhood of $\mathbf{p}$, has distinct images $U(\mathbf{p}+\mathbf{d})$ in (a) and (b); in the first case, the digitization operator $\mathcal{D}$ maps $\mathcal{U}(\mathbf{p}+\mathbf{d})$ onto the blue integer point, while in the second case, it maps it onto the red one.

of its neighbors with respect to $U$ may also change (see Figure 4). In other words, the neighborhood motion map $\mathcal{G}_{r}^{U}(\mathbf{p})$ evolves non-continuously according to the parameters of $\mathcal{U}$ that underlies $U$. Our purpose is now to express how $\mathcal{G}_{r}^{U}(\mathbf{p})$ evolves.
First of all, it is important to remark that the continuous image $\mathcal{U}(\mathbf{p})$ and the digital image $U(\mathbf{p})$ of $\mathbf{p}$ are spatially linked by the digitization operator $\mathcal{D}$. More precisely, from Equation (3) we have

$$
\begin{aligned}
& \mathcal{U}(\mathbf{p}) \in C(U(\mathbf{p}))= \\
& {\left[p_{1}-\frac{1}{2}, p_{1}+\frac{1}{2}\right) \times\left[p_{2}-\frac{1}{2}, p_{2}+\frac{1}{2}\right)}
\end{aligned}
$$

where $U(\mathbf{p})=\left(p_{1}, p_{2}\right) \in \mathbb{Z}^{2}$. In other words, the continuous image $\mathcal{U}(\mathbf{p})$ lies within the unit square $C(U(\mathbf{p}))$ - the digitization cell-of $\mathbb{R}^{2}$ whose center is the integer point $U(\mathbf{p})$ of $\mathbb{Z}^{2}$, and we define $\rho(\mathbf{p})=\mathcal{U}(\mathbf{p})-U(\mathbf{p})$, taking values in $\mathscr{P}=\left[-\frac{1}{2}, \frac{1}{2}\right)^{2}$. Note that $\rho(\mathbf{p})$ depends on $\left(t_{x}, t_{y}, \theta\right)$. The coordinates of $\rho(\mathbf{p})$, called the remainder of $\mathbf{p}$ under $\mathcal{U}$, are the fractional part of the coordinates of $\mathcal{U}(\mathbf{p})$, and $\rho$ is the remainder map under $U$. The range of $\rho, \mathscr{P}=\left[-\frac{1}{2}, \frac{1}{2}\right)^{2}$, is called the remainder range.

Let us now consider an integer point $\mathbf{p}+\mathbf{d}$ in the neighborhood $\mathcal{N}_{r}(\mathbf{p})$ of $\mathbf{p}$. From Equation (1), we have

$$
\mathcal{U}(\mathbf{p}+\mathbf{d})=\mathbf{R d}+\mathcal{U}(\mathbf{p}) .
$$

We may rewrite it as $\mathcal{U}(\mathbf{p}+\mathbf{d})=\mathbf{R d}+\rho(\mathbf{p})+U(\mathbf{p})$. Without loss of generality - and up to translations in $\mathbb{Z}^{2}$-we can assume that $U(\mathbf{p})$ is the origin of a local coordinate frame of the image space, i.e. $\mathcal{U}(\mathbf{p}) \in \mathscr{P}$. In these local coordinates frames, the former equation rewrites as

$\mathcal{U}(\mathbf{p}+\mathbf{d})=\mathbf{R d}+\rho(\mathbf{p})$.

Now, studying the non-continuous evolution of the neighborhood motion map $\mathcal{G}_{r}^{U}(\mathbf{p})$ is equivalent to studying the behavior of $U(\mathbf{p}+\mathbf{d})=\mathcal{D} \circ \mathcal{U}(\mathbf{p}+\mathbf{d})$ for $\mathbf{d} \in \mathcal{N}_{r}(\mathbf{0})$ and $\mathcal{U}(\mathbf{p}) \in \mathscr{P}$, with respect to the rotation parameter $\theta$ defining $\mathbf{R}$ and the translation parameters embedded by $\rho(\mathbf{p})$, that deterministically depend on $\left(t_{x}, t_{y}, \theta\right)$. In particular, the discontinuities of $U(\mathbf{p}+\mathbf{d})$ occur when $\mathcal{U}(\mathbf{p}+\mathbf{d})$ is on the boundary of a digitization cell, as illustrated in Figures 4 and 5. Setting $\rho(\mathbf{p})=(x, y) \in \mathscr{P}$ and $\mathbf{d}=(u, v) \in \mathcal{N}_{r}(\mathbf{0})$, this is formulated by one of the following two equations

$$
\begin{aligned}
& x+u \cos \theta-v \sin \theta=k_{x}+\frac{1}{2} \\
& y+u \sin \theta+v \cos \theta=k_{y}+\frac{1}{2}
\end{aligned}
$$




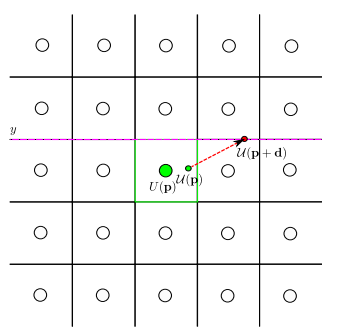

(a)

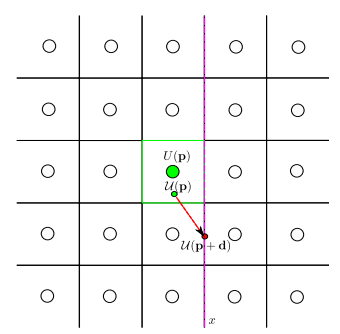

(b)
Fig. 5 Critical cases: $\mathcal{U}(\mathbf{p}+\mathbf{d})$ is located on (a) a horizontal, and (b) a vertical boundary of a digitization cell.

where $k_{x}, k_{y} \in \mathbb{Z}$. For given $\mathbf{d}=(u, v)$ and $k_{x}$ (resp. $k_{y}$ ), Equation (6) (resp. (7)) defines a vertical (resp. horizontal) line in the remainder range $\mathscr{P}$, called a vertical (resp. horizontal) critical line. These critical lines with different $\mathbf{d}, k_{x}$ and $k_{y}$ subdivide the remainder range $\mathscr{P}$ into rectangular regions called frames. As long as coordinates of $\rho(\mathbf{p})$ belong to a same frame, the associated neighborhood motion map $\mathcal{G}_{r}^{U}(\mathbf{p})$ remains constant.

Proposition 1 For any $\mathbf{p}, \mathbf{q} \in \mathbb{Z}^{2}, \mathcal{G}_{r}^{U}(\mathbf{p})=\mathcal{G}_{r}^{U}(\mathbf{q})$ iff $\rho(\mathbf{p})$ and $\rho(\mathbf{q})$ are in the same frame.

A similar proposition was already shown in [6] for the case $r=1$ and rotations. The above result is then an extension for general cases, such that $r \geq 1$, and rigid motions. An example of the remainder range partitioning is presented in Figure 6.

Remark 3 Equations (6) and (7) of critical lines are similar to those for digitized rotations, since the translation part is embedded only in $\rho(\mathbf{p})=(x, y)$, as seen in Equation (5).

In digital topology and geometry, the following two neighborhoods play a special role: $\mathcal{N}_{1}(\mathbf{p})$ and $\mathcal{N}_{2}(\mathbf{p})$. Therefore, in the following parts of this section, we extend the work of Nouvel and Rémila [6], by considering not only digitized rigid motions but also both $\mathcal{N}_{1}(\mathbf{p})$ and $\mathcal{N}_{2}(\mathbf{p})$. The study of the neighborhood motion maps for $\mathcal{N}_{1}(\mathbf{p})$ and $\mathcal{N}_{2}(\mathbf{p})$ will allow us, later on, to observe non-injective and non-surjective motions. It should be emphasized that non-surjectivity can be seen only when $\mathcal{N}_{2}(\mathbf{p})$ is considered. In particular, $\mathcal{N}_{1}(\mathbf{p})$ is not suffciently large for this observation (see Section 3.5 for details).

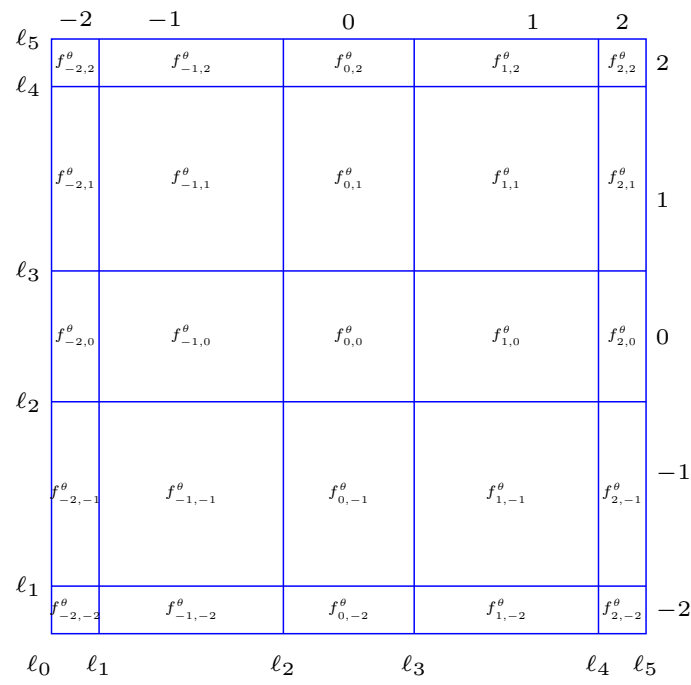

(a)

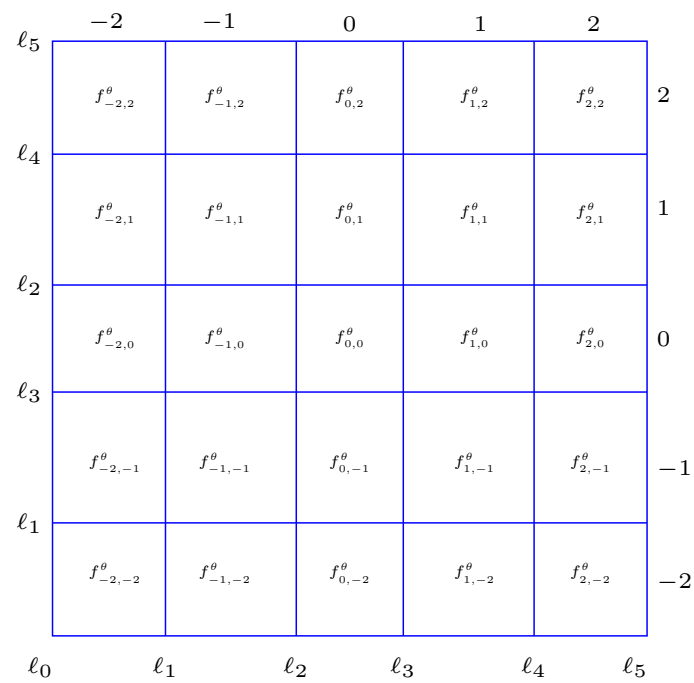

(b)

Fig. 6 Frames of the remainder range $\mathscr{P}$ for a given $\theta$ : (a) $0<\theta<\frac{\pi}{6}$ and (b) $\frac{\pi}{6}<\theta<\frac{\pi}{4}$.

Thanks to angular symmetries by $\frac{\pi}{4}$, and based on the above discussion, we can restrict, without loss of generality, the parameter space of $\left(t_{x}, t_{y}, \theta\right)$ to $\left[-\frac{1}{2}, \frac{1}{2}\right)^{2} \times\left[0, \frac{\pi}{4}\right)$. The set of all rigid motions $\mathcal{U}$ which belong to the restricted parameter space is denoted by $\mathbb{U}$. 


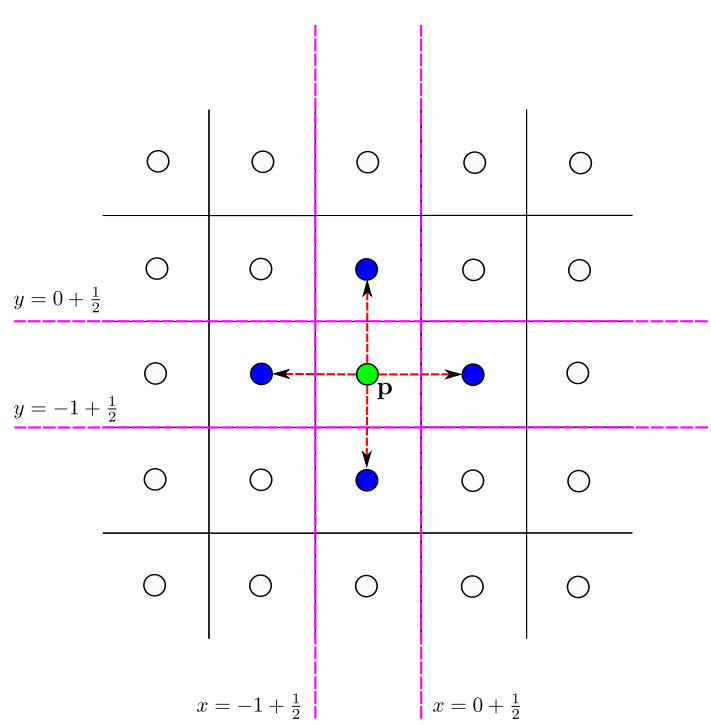

Fig. 7 Vertical and horizontal lines $x=k_{x}+\frac{1}{2}, y=$ $k_{y}+\frac{1}{2}$ where $k_{x}, k_{y} \in\{-1,0\}$-in pink-for $\mathbf{d}=(u, v)=$ $(0,0),( \pm 1,0),(0, \pm 1)$, where $\mathbf{p}$ is depicted in green and $\mathbf{p}+\mathbf{d}, \mathbf{d} \neq(0,0)$ in blue. For $\mathbf{d}=(1,0)$, we have $k_{x}=0, k_{y}=$ 0 ; for $\mathbf{d}=(0,1), k_{x}=-1, k_{y}=0$; for $\mathbf{d}=(-1,0), k_{x}=$ $-1, k_{y}=-1 ;$ and for $\mathbf{d}=(0,-1), k_{x}=0, k_{y}=-1$.

$3.2 \mathcal{N}_{1}$ neighborhood case: $\mathcal{G}_{1}^{U}$

In accordance with critical lines (Equations (67)), a partition of $\mathscr{P}$ for $\mathcal{N}_{1}(\mathbf{p})$ is obtained from $\mathbf{d}=(u, v)=(0,0),( \pm 1,0)$ or $(0, \pm 1)$ and $k_{x}, k_{y} \in$ $\{-1,0\}$. Critical lines for each $\mathbf{d}$ depend on gridlines: $x=k_{x}+\frac{1}{2}, y=k_{y}+\frac{1}{2}$ whose neighbor $\mathbf{p}+\mathbf{d}$ can cross under $\mathcal{U}$. In case of $\mathcal{N}_{1}(\mathbf{p})$ and $\mathcal{U} \in \mathbb{U}$, for $\mathbf{d}=(0,0)$, we have two vertical and two horizontal lines, while for $\mathbf{d}=( \pm 1,0)$ (resp. $(0, \pm 1))$, we have one vertical (resp. horizontal) line, as presented in Figure 7. Vertical critical lines defined for different values of $\mathbf{d}$ and $k_{x}$ are given in Table 1, while the horizontal lines can be obtained by replacing $x$ with $y$. For this reason, we restrict our following discussion to vertical critical lines.

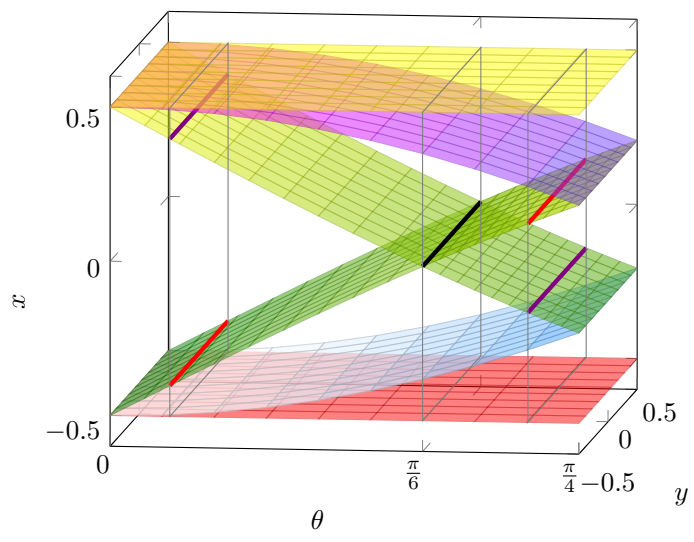

Fig. 8 Partitions of the remainder range $\mathscr{P}$ by vertical critical lines for $0<\theta<\frac{\pi}{4}$. For two different values of $\theta$, lines $\ell_{2}$ and $\ell_{3}$ are depicted in purple and red, respectively. For $\theta=\frac{\pi}{6}$, the lines $\ell_{2}$ and $\ell_{3}$ are equal. The intersection of surfaces which represents this situation is marked by a black line.

\begin{tabular}{|c|l|c|c|}
\hline Line & Equation & $\mathbf{d}$ & $k_{x}$ \\
\hline$\ell_{0}$ & $x=-\frac{1}{2}$ & $(0,0)$ & -1 \\
\hline$\ell_{1}$ & $x=\frac{1}{2}-\cos \theta$ & $(1,0)$ & 0 \\
\hline$\ell_{2}$ & $x=\sin \theta-\frac{1}{2}$ & $(0,1)$ & -1 \\
\hline$\ell_{3}$ & $x=\frac{1}{2}-\sin \theta$ & $(0,-1)$ & 0 \\
\hline$\ell_{4}$ & $x=\cos \theta-\frac{1}{2}$ & $(-1,0)$ & -1 \\
\hline$\ell_{5}$ & $x=\frac{1}{2}$ & $(0,0)$ & 0 \\
\hline
\end{tabular}

Table 1: Vertical critical lines for $\mathcal{N}_{1}(\mathbf{p})$.

It is worth mentioning that critical lines change their positions depending on $\theta$. When $0<$ $\theta<\frac{\pi}{6}$, their order with respect to the $x$-axis is $\left(\ell_{0}, \ell_{1}, \ell_{2}, \ell_{3}, \ell_{4}, \ell_{5}\right)$. Then, when $\theta=\frac{\pi}{6}$, lines $\ell_{2}$ and $\ell_{3}$ merge, and for $\theta>\frac{\pi}{6}$ their order is reversed. This situation is illustrated by Figure 8 . Notice that this is the only order change for $0<\theta<\frac{\pi}{4}$. As $\ell_{0}$ and $\ell_{5}$ are on the border of the remainder range and do not move, the vertical lines subdivide the remainder range into 5 parts, expect for $\theta=\frac{\pi}{6}$.

At some $\theta$, suppose the orders of vertical and horizontal critical lines are $\left(\ell_{1}^{v}, \ldots, \ell_{n}^{v}\right)$ and $\left(\ell_{1}^{h}, \ldots, \ell_{n}^{h}\right)$, respectively. Then, the frame bounded by $\ell_{i}^{v}, \ell_{i+1}^{v}, \ell_{j}^{h}, \ell_{j+1}^{h}$ has the index $\left(i-\frac{n}{2}, j-\frac{n}{2}\right)$; this frame is then noted $f_{i-\frac{n}{2}, j-\frac{n}{2}}^{\theta}$, and it is defined as

$$
f_{i-\frac{n}{2}, j-\frac{n}{2}}^{\theta}=\left[\ell_{i}^{v}(\theta), \ell_{i+1}^{v}(\theta)\right) \times\left[\ell_{j}^{h}(\theta), \ell_{j+1}^{h}(\theta)\right),
$$




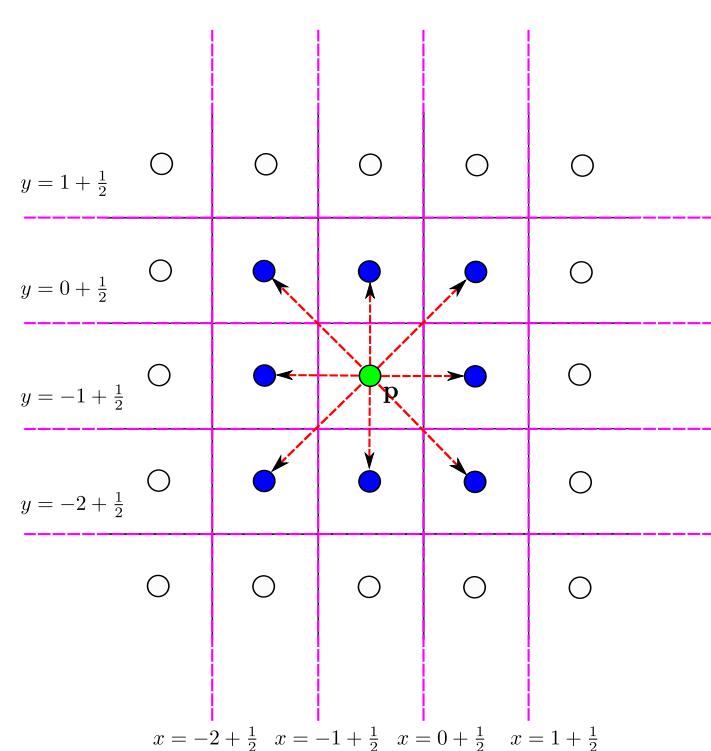

Fig. 9 Vertical and horizontal lines $x=k_{x}+\frac{1}{2}, y=k_{y}+\frac{1}{2}$ where $k_{x}, k_{y} \in\{-2,-1,0,1\}$-in pink-for $\mathbf{d}=(u, v) \stackrel{2}{=}$ $(0,0),( \pm 1,0),(0, \pm 1),( \pm 1, \pm 1)$, where $\mathbf{p}$ is depicted in green and $\mathbf{p}+\mathbf{d}, \mathbf{d} \neq(0,0)$ in blue. For $\mathbf{d}=(1,1)$, we, have $k_{x}=0, k_{y}=1$; for $\mathbf{d}=(-1,1), k_{x}=-2, k_{y}=0$; for $\mathbf{d}=$ $(-1,-1), k_{x}=-1, k_{y}=-2$; for $\mathbf{d}=(1,-1), k_{x}=1, k_{y}=-1$.

where $\ell_{i}^{v}(\theta)$ (resp. $\left.\ell_{j}^{h}(\theta)\right)$ denotes the $x$ (resp. $y$ ) coordinate of the line $\ell_{i}^{v}$ (resp. $\ell_{j}^{h}$ ) for given $\theta$. Based on such an index definition, a frame of the indices $(0,0)$ is located at the center of the remainder range, which allows for rapid and easy identification of symmetric neighborhood motion maps, as detailed later on. Partitions of the remainder range $\mathscr{P}$ for $\theta \in\left(0, \frac{\pi}{6}\right)$ and $\theta \in\left(\frac{\pi}{6}, \frac{\pi}{4}\right)$ are presented in Figure 6 .

\section{$3.3 \mathcal{N}_{2}$ neighborhood case: $\mathcal{G}_{2}^{U}$}

In the case of $\mathcal{N}_{2}(\mathbf{p})$, a partition of the remainder range $\mathscr{P}$ is obtained in a similar way as for $\mathcal{N}_{1}(\mathbf{p})$, from the different values: d $\in\{(0,0),(0, \pm 1),( \pm 1,0),( \pm 1, \pm 1)\}$ and $k_{x}, k_{y} \in$ $\{-2,-1,0,1\}$ (see Figure 9). Notice that, as $\mathcal{N}_{1}(\mathbf{p}) \subset \mathcal{N}_{2}(\mathbf{p})$, some of the critical lines for $\mathcal{N}_{2}(\mathbf{p})$ are the same as for $\mathcal{N}_{1}(\mathbf{p})$. The supplementary vertical critical lines for the values of $\mathbf{d} \in \mathcal{N}_{2}(\mathbf{0}) \backslash \mathcal{N}_{1}(\mathbf{0})$ are given in Table 2, while the horizontal lines can be obtained by replacing $x$ with $y$.

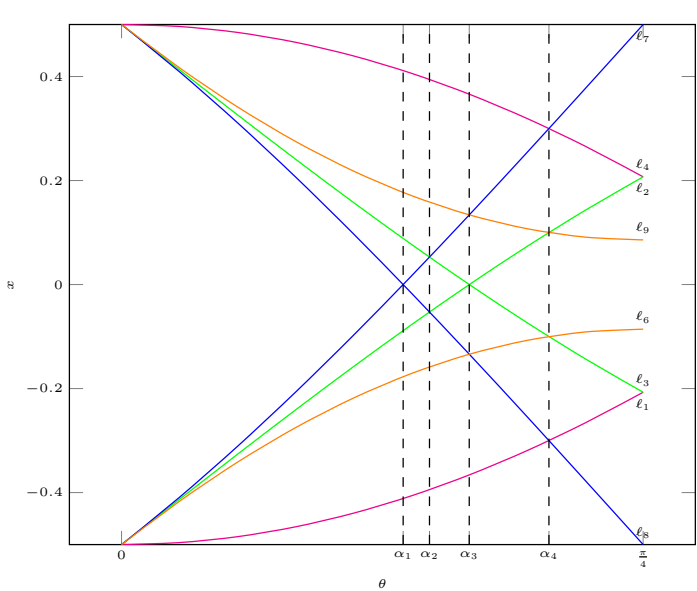

Fig. 10 Partition of the remainder range $\mathscr{P}$ by vertical critical lines for $\mathcal{N}_{2}(\mathbf{p})$ and $0<\theta<\frac{\pi}{4}$. In pink: $\ell_{1}$ and $\ell_{4}$; in green: $\ell_{2}$ and $\ell_{3}$; in blue: $\ell_{7}$ and $\ell_{8}$; in orange: $\ell_{6}$ and $\ell_{9}$. For better visualization, critical lines are projected on the $(x, \theta)$-plane.

\begin{tabular}{|c|l|c|c|}
\hline Line & Equation & d & $k_{x}$ \\
\hline$\ell_{6}$ & $x=(\cos \theta+\sin \theta)-\frac{3}{2}$ & $(-1,1)$ & -2 \\
\hline$\ell_{7}$ & $x=\frac{1}{2}-(\cos \theta-\sin \theta)$ & $(1,1)$ & 0 \\
\hline$\ell_{8}$ & $x=\cos \theta-\sin \theta-\frac{1}{2}$ & $(-1,-1)$ & -1 \\
\hline$\ell_{9}$ & $x=\frac{3}{2}-(\cos \theta+\sin \theta)$ & $(1,-1)$ & 1 \\
\hline
\end{tabular}

Table 2: Vertical critical lines for $\mathcal{N}_{2}(\mathbf{p})$, which are defined for $\mathbf{p}+\mathbf{d} \in \mathcal{N}_{2}(\mathbf{p}) \backslash \mathcal{N}_{1}(\mathbf{p})$.

From Figure 10, we can notice that, for $\theta \in$ $\left(0, \frac{\pi}{4}\right)$ we have to consider four special angles, each inducing change of the order of critical lines. In particular, for $\alpha_{n-1}<\theta<\alpha_{n}, n=1,2,3,4,5$ with $\alpha_{0}=0$ and $\alpha_{5}=\frac{\pi}{4}$, the order of critical lines is constant and initially equal to $\left(\ell_{0}, \ell_{1}, \ell_{6}, \ell_{2}\right.$, $\left.\ell_{7}, \ell_{8}, \ell_{3}, \ell_{9}, \ell_{4}, \ell_{5}\right)$.

\subsection{Set of neighborhood motion maps}

In the preceding sections, we have seen that critical lines subdivide the remainder range for any $\theta$ into a finite number of frames. From Proposition 1, we can then observe the set of all distinct neighborhood motion maps:

$$
\mathbb{Q}_{r}=\bigcup_{\mathcal{U} \in \mathbb{U}} \bigcup_{\mathbf{p} \in \mathbb{Z}^{2}}\left\{\mathcal{G}_{r}^{U}(\mathbf{p})\right\}
$$


where $\mathbb{U}$ is the set of all rigid motions $\mathcal{U}$ defined by the restricted parameter space $\left(-\frac{1}{2}, \frac{1}{2}\right]^{2} \times\left[0, \frac{\pi}{4}\right)$.

The cardinality of $\mathbb{Q}_{r}$ for $r=1$ is equal to 34 , while for $r=2$, it is equal to 231. It should be also noticed that $\left|\bigcup_{\mathbf{p} \in \mathbb{Z}^{2}}\left\{\mathcal{G}_{r}^{U}(\mathbf{p})\right\}\right|$ is constant: 25 when $r=1,81$ when $r=2$, for any $\mathcal{U}$, except for some special rotation angles. For instance, we have $\left|\bigcup_{\mathbf{p} \in \mathbb{Z}^{2}}\left\{\mathcal{G}_{1}^{U}(\mathbf{p})\right\}\right|=1$ for $\theta=0,16$ for $\theta=\frac{\pi}{6}$, and 9 for $\theta=\frac{\pi}{4}$.

Such elements of sets $\mathbb{Q}_{1}$ and $\mathbb{Q}_{2}$ are presented in Appendix A and Appendix B, respectively. Each neighborhood motion map given in Appendices can be identified thanks to indices of associated frames of the remainder range. We can remark that neighborhood motion maps are symmetric with respect to the origin - the frame of indices $(0,0)$. For example, the neighborhood motion map of indices $(-3,4)$ is symmetric to that of the indices $(3,-4)$ (see Appendix B, Figure 20).

\subsection{Non-surjective and non-injective frames}

Some frames correspond to neighborhood motion maps that have points with two or zero preimages, implying non-surjectivity or non-injectivity [7].

Neighborhood motion maps which present nonsurjectivity can be found in Appendix B. They possess at least one non-labeled point $\mathbf{w}$ (white square) that is surrounded by four labeled points at $\mathcal{N}_{1}(\mathbf{w})$, whose preimages form a $2 \times 2$ square (see the neighborhood motion map of the frame $f_{0,0}^{\theta}$ for their preimages). For example, see the frames $(2,-3),(3,-3),(2,-4)$ and $(3,-4)$, depicted in Figure 20 .

Lemma $1 U(\mathbf{p})+\mathbf{d}_{*}$ has no preimage if and only if $\rho(\mathbf{p})$ is in one of the zones $f_{*}^{0}$ (union of frames themselves) defined as follows:

$$
\begin{aligned}
f_{\uparrow}^{0} & =\left[\frac{1}{2}-\cos \theta, \sin \theta-\frac{1}{2}\right) \times\left[\frac{3}{2}-\cos \theta-\sin \theta, \frac{1}{2}\right), \\
f_{\rightarrow}^{0} & =\left[\frac{3}{2}-\cos \theta-\sin \theta, \frac{1}{2}\right) \times\left[\frac{1}{2}-\sin \theta, \cos \theta-\frac{1}{2}\right), \\
f_{\downarrow}^{0} & =\left[\frac{1}{2}-\sin \theta, \cos \theta-\frac{1}{2}\right) \times\left[-\frac{1}{2}, \cos \theta+\sin \theta-\frac{3}{2}\right), \\
f_{\leftarrow}^{0} & =\left[-\frac{1}{2}, \cos \theta+\sin \theta-\frac{3}{2}\right) \times\left[\frac{1}{2}-\cos \theta, \sin \theta-\frac{1}{2}\right),
\end{aligned}
$$

where $* \in\{\uparrow, \rightarrow, \downarrow, \leftarrow\}$ and $\mathbf{d}_{\uparrow}=(0,1), \mathbf{d}_{\rightarrow}=$ $(1,0), \mathbf{d}_{\downarrow}=(0,-1), \mathbf{d}_{\leftarrow}=(-1,0)$.

The non-surjective zones are defined by three critical lines given by $\mathcal{N}_{1}(\mathbf{0})$ and one given by $\mathcal{N}_{2}(\boldsymbol{0})$. For instance, the zone $f_{\uparrow}^{0}$ is given by the vertical lines $\ell_{1}$ and $\ell_{2}$ and the horizontal lines $\ell_{9}$ and $\ell_{5}$.

Neighborhood motion maps which present noninjectivity can be observed in Appendix A. They have two labels at the center. For instance, see the frames $(2,2),(2,1)$ and $(2,0)$ in Figure 18.

Lemma $2 U(\mathbf{p})$ has two preimages which are $\mathbf{p}$ and $\mathbf{p}+\mathbf{d}_{*}$ if and only if $\rho(\mathbf{p})$ is in one of the zones $f_{*}^{2}$ defined as follows:

$$
\begin{aligned}
& f_{\uparrow}^{2}=\left[\sin \theta-\frac{1}{2}, \frac{1}{2}\right) \times\left[-\frac{1}{2}, \frac{1}{2}-\cos \theta\right), \\
& f_{\rightarrow}^{2}=\left[-\frac{1}{2}, \frac{1}{2}-\cos \theta\right) \times\left[-\frac{1}{2}, \frac{1}{2}-\sin \theta\right), \\
& f_{\downarrow}^{2}=\left[-\frac{1}{2}, \frac{1}{2}-\sin \theta\right) \times\left[\cos \theta-\frac{1}{2}, \frac{1}{2}\right), \\
& f_{\leftarrow}^{2}=\left[\cos \theta-\frac{1}{2}, \frac{1}{2}\right) \times\left[\sin \theta-\frac{1}{2}, \frac{1}{2}\right) .
\end{aligned}
$$

We can characterize the non-surjectivity and non-injectivity of a digitized rigid motion by the presence of $\rho(\mathbf{p})$ in these specific zones. Both types of zones are presented in Figure 11.

\section{Globally bijective digitized rigid motions}

A digitized rigid motion is bijective if and only if there is no $\rho(\mathbf{p})$ for all $\mathbf{p} \in \mathbb{Z}^{2}$ in non-surjective and non-injective zones of $\mathscr{P}$. In this section, we characterize bijective rigid motions on $\mathbb{Z}^{2}$ while investigating such local conditions.

Let us start with the rotational part of the motion. We know from [7] that rotations with any angle of irrational sine or cosine are non-bijective; indeed, such rotations have a dense image by $\rho$ (there exists $\mathbf{p} \in \mathbb{Z}^{2}$ such that $\rho(\mathbf{p})$ lies in a nonsurjective and/or non-injective zone of $\mathscr{P}$ ). This result is also applied to $U$, whatever translation part is added.

Therefore, we focus on rigid motions for which both cosine and sine of the angle $\theta$ are rational. Such angles are called Pythagorean angles [7] 


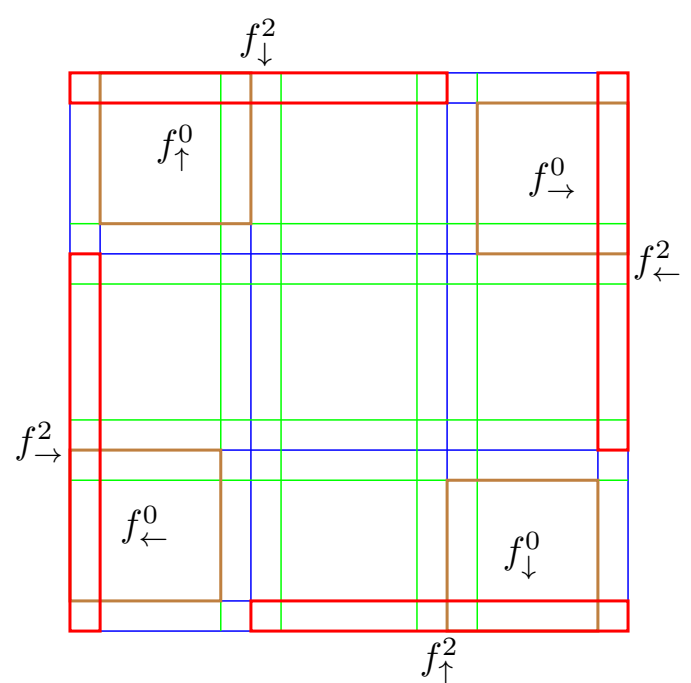

Fig. 11 Example of remainder range partitioning for $r=$ 2 together with non-injective zones $f_{*}^{2}$ and non-surjective zones $f_{*}^{0}$ which are illustrated by red and brown rectangles, respectively.

and are defined by primitive Pythagorean triples $(a, b, c)=\left(p^{2}-q^{2}, 2 p q, p^{2}+q^{2}\right)$ with $p, q \in \mathbb{Z}, p>$ $q$ and $p-q$ is odd, such that $(a, b, c)$ are pairwise coprime, and cosine and sine of such angles are $\frac{a}{c}$ and $\frac{b}{c}$, respectively. The image of $\mathbb{Z}^{2}$ by $\rho$, when $U$ is a digitized rational rotation, corresponds to a cyclic group $\mathcal{G}$ on the remainder range $\mathscr{P}$, which is generated by $\boldsymbol{\psi}=\left(\frac{p}{c}, \frac{q}{c}\right)$ and $\boldsymbol{\omega}=\left(-\frac{q}{c}, \frac{p}{c}\right)$ and whose order is equal to $c=p^{2}+q^{2}$ [7]. When $U$ contains a translation part, the image of $\rho$ in $\mathscr{P}$, which we denote by $\mathcal{L}^{\prime}$, is obtained by translating $\mathscr{G}_{\mathcal{L}}\left(\right.$ modulo $\mathbb{Z}^{2}$ ), and $\left|\mathcal{L}^{\prime}\right|$ is equal to the order of $\mathscr{L}_{\mathcal{L}}$, its underlying group. Note also that a digitized rational rotation is bijective (the intersection of $\mathcal{G}_{\mathcal{L}}$ with non-injective and non-surjective regions is empty) iff its angle comes from a twin Pythagorean triplea primitive Pythagorean triple with the additional condition $p=q+1$-see Nouvel and Rémila [7] and, more recently, Roussillon and Cœurjolly [12].

Our question is then whether a digitized rigid motion can be bijective, even when the corresponding rotation is not. In order to answer this question, we use the following equivalence property: digitized rational rotations are bijective if they are surjective or injective [7]. Indeed, this allows us to focus only on non-surjective zones; since they are squares, they provide symmetry and then present interesting properties in terms of exact computing.

Proposition 2 A digitized rigid motion whose rotational part is given by a non-twin Pythagorean primitive triple is always non-surjective.

Proof We show that no translation factor can prevent the existence of an element of $\mathcal{L}^{\prime}$ in a nonsurjective zone. We consider the length of a side of $f_{*}^{0}$, given by $L_{1}=\frac{2 q(p-q)}{c}$, and the side of the bounding box of a fundamental square in $\mathscr{L}_{\mathscr{L}}$, given by $L_{2}=\frac{p+q}{c}$. Note that any non-surjective zone $f_{*}^{0}$ also forms a square. Then by comparing $L_{1}$ with $L_{2}$, we have that, as $p>q+1, L_{2}<L_{1}$, and thus $\mathcal{G}^{\prime} \cap f_{*}^{0} \neq \varnothing$ (see Figure 12(a)).

If, on the contrary, the rotational part is given by a twin Pythagorean triple, i.e. is bijective, then the rigid motion is also bijective, under the following condition.

Proposition 3 A digitized rigid motion is bijective if and only if it is composed of a rotation by an angle defined by a twin Pythagorean triple and a translation $\mathbf{t}=\mathbf{t}^{\prime}+\mathbb{Z} \boldsymbol{\psi}+\mathbb{Z} \boldsymbol{\omega}$, where $\mathbf{t}^{\prime} \in\left(-\frac{1}{2 c}, \frac{1}{2 c}\right)^{2}$.

Proof Let us first consider the case $\mathbf{t}=\mathbf{0}$. Since $L_{2}>L_{1}$, there exists a fundamental square in $\mathscr{L}_{\text {, i.e. }}$ whose vertices are $(n \boldsymbol{\omega}+m \boldsymbol{\psi}),((n+1) \boldsymbol{\omega}+m \boldsymbol{\psi}),((n+$ $1) \boldsymbol{\omega}+(m+1) \boldsymbol{\psi}),(n \boldsymbol{\omega}+(m+1) \boldsymbol{\psi})$, where $n, m \in \mathbb{Z}$, and the vertices lie outside of $f_{\downarrow}^{0}$, at $N_{\infty}$ distance $\frac{1}{2 c}$ (see Figure 12(b) and the proof of Theorem 5 in [7]). Now, let us consider the case $\mathbf{t} \neq \mathbf{0}$. The above four vertices are the elements of $\mathcal{G}_{\mathcal{C}}$ closest to $f_{\downarrow}^{0}$, therefore if $N_{\infty}(\{\mathbf{t}\})<\frac{1}{2 c}$, where $\{$.$\} stands$ for the fractional part function, then $\mathcal{L}^{\prime} \cap f_{\downarrow}^{0}=\varnothing$. Moreover, if $N_{\infty}(\{\mathbf{t}\})$ is slightly above $\frac{1}{2 c}$, then it is plain that some point of $\mathcal{G}^{\prime}$ will enter the frame $f_{\downarrow}^{0}$. But $\mathcal{G}$ is periodic with periods $\omega$ and $\psi$, so that the set of admissible vectors $\mathbf{t}$ has the same periods. Then, we see that the admissible vectors form a square (i.e. a $N_{\infty}$ ball of radius $\frac{1}{2 c}$ ) modulo $\mathbb{Z} \boldsymbol{\psi}+\mathbb{Z} \boldsymbol{\omega}$ (see Figure 12(c)) 


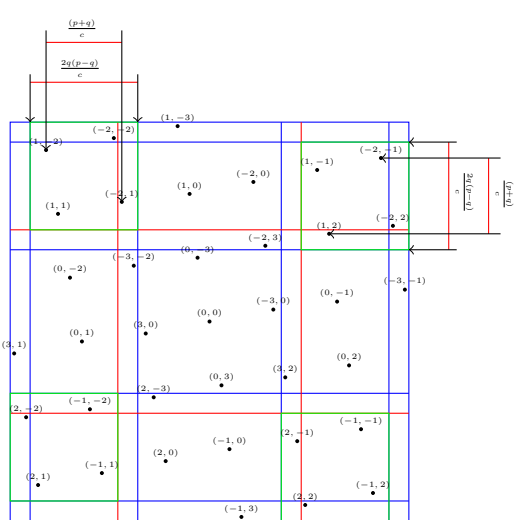

(a)

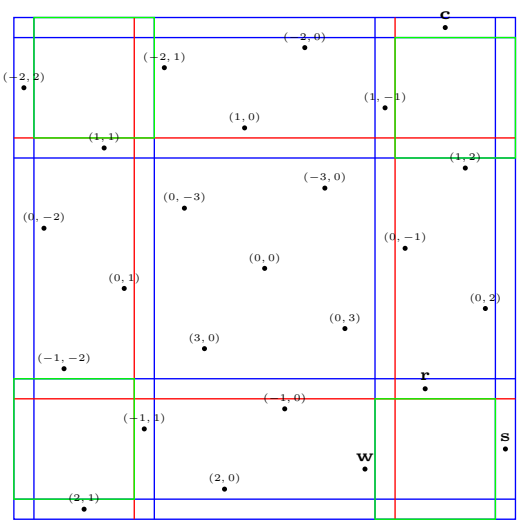

(b)

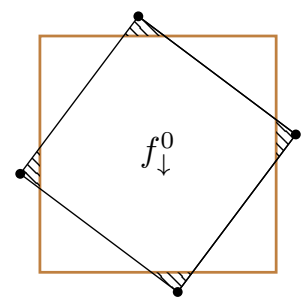

(c)

Fig. 12 Examples of remainder range partitioning together with $\mathcal{C}_{\mathcal{L}}$ obtained for rotations by Pythagorean angles. (a) The non-bijective digitized rotation defined by the primitive Pythagorean triple $(12,35,37)$, and (b) the bijective digitized rotation defined by the twin Pythagorean triple $(7,24,25)$. The non-surjective and non-injective zones are illustrated by brown and red rectangles, respectively. (c) A fundamental square in $\mathcal{L}$ whose vertices are $(n \boldsymbol{\omega}+m \boldsymbol{\psi}),((n+1) \boldsymbol{\omega}+$ $m \boldsymbol{\psi}),((n+1) \boldsymbol{\omega}+(m+1) \boldsymbol{\psi}),(n \boldsymbol{\omega}+(m+1) \boldsymbol{\psi})$, represented by black circles, and $f^{0}$ in brown. The union of the areas filled with a line pattern forms a square (i.e. a $N_{\infty}$ ball of radius $\frac{1}{2 c}$ ) of the admissible translation vectors modulo $\mathbb{Z} \boldsymbol{\psi}+\mathbb{Z} \boldsymbol{\omega}$.

\section{Locally bijective digitized rigid motions}

As seen above, the bijective digitized rigid motions, though numerous, are not dense in the set of all digitized rigid motions. Thus, we may generally expect defects, such as points with two preimages. However, in practical applications, the bijectivity of a given $U$ on the whole $\mathbb{Z}^{2}$ is not the main issue; rather, one usually works on a finite subset of the plane (e.g., a square digital image). The relevant question is then: "given a finite subset $S \subset \mathbb{Z}^{2}$, is $U$ restricted to $S$ bijective?". Actually, the notion of bijectivity in this question can be replaced by the notion of injectivity, since the surjectivity is trivial, due to the definition of $U$ that maps $S$ to $U(S)$.

The basic idea for such local bijectivity verification is quite natural. Because of its quasi-isometric property, a digitized rigid motion $U$ can send at most two 4-neighbors onto a same point. Thus, the lack of injectivity is a purely local matter, suitably handled by the neighborhood motion maps via the remainder map. Indeed, in accordance with Lemma 2, $U$ is non-injective, with respect to $S$ iff there exists $\mathbf{p} \in S$ such that $\rho(\mathbf{p})$ lies in the union $\mathcal{F}=f_{\downarrow}^{2} \cup f_{\uparrow}^{2} \cup f_{\leftarrow}^{2} \cup f_{\rightarrow}^{2}$ of all non-injective zones. We propose two algorithms making use of the remainder map information, as an alternative to a brute force verification.

The first-forward - algorithm, verifies for each point $\mathbf{p} \in S$, the inclusion of $\rho(\mathbf{p})$ in one of the non-injective zones of $\mathcal{F}$. The second-backwardalgorithm first finds all points $\mathbf{w}$ in $\mathcal{L}^{\prime} \cap \mathcal{F}$, called the non-injective remainder set, and then verifies if their preimages $\rho^{-1}(\mathbf{w})$ are in $S$.

Both algorithms apply to rational motions, i.e., with a Pythagorean angle given by a primitive Pythagorean triple $(a, b, c)=\left(p^{2}-q^{2}, 2 p q, p^{2}+q^{2}\right)$ and a rational translation vector $\mathbf{t}=\left(t_{x}, t_{y}\right)$. We capture essentially the behavior for all angles and translation vectors, since rational motions are dense. These assumptions guarantee the exact computations of the algorithms, which are based on integer numbers. Methods for angle approximation by Pythagorean triples up to a given precision may be found in [1]. 


\subsection{The forward algorithm}

The strategy consists of checking whether the remainder map $\rho(\mathbf{p})$ of each $\mathbf{p} \in S$ belongs to one of the non-injective zones $f_{*}^{2}$ defined in Lemma 2; if this is the case, we check additionally whether $\mathbf{p}+\mathbf{d}_{*} \in \mathcal{N}_{1}(\mathbf{p})$ belongs to $S$; otherwise, there is no non-injective mapping with $\mathbf{p}$ under $U_{\mid S}$.

This leads to the forward algorithm, which returns the set $B$ of all pairs of points having the same image. We can then conclude that $U_{\mid S}$ is bijective iff $B=\varnothing$; in other words, $U$ is injective on $S \backslash B$. The break statement on line 7 comes from the fact that, in accordance with Remark 1, a 2D integer point can have at most two preimages. Using the same argument, we also restrict the internal loop to the set $\{\rightarrow, \downarrow\}$.

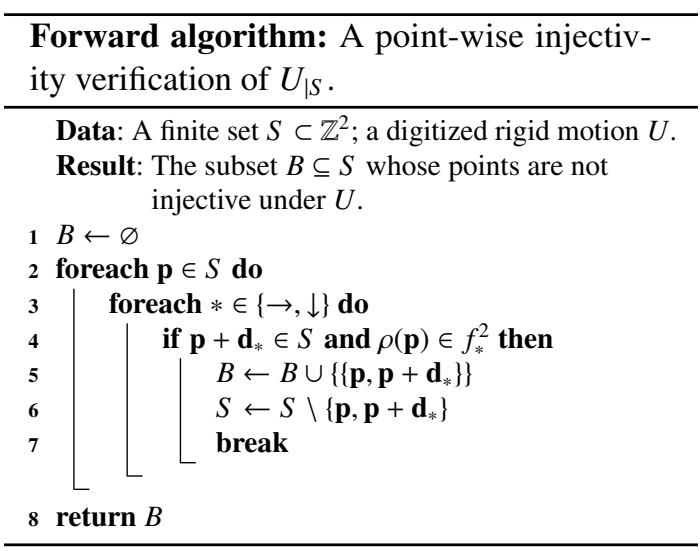

The main advantage of the forward algorithm lies in its simplicity. In particular, we can directly check which neighbor $\mathbf{p}+\mathbf{d}_{*}$ of $\mathbf{p}$ has the same image under a digitized rigid motion. Because rational rigid motions are exactly represented by integers, it can be verified without numerical error and in constant time, if $\rho(\mathbf{p}) \in \mathcal{F}$. The time complexity of this algorithm is $\mathcal{O}(|S|)$. Figure 13 illustrates the forward algorithm.

Remark 4 The forward algorithm can be used with non-rational rigid motions, at the cost of a numerical error.

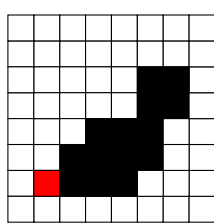

(a)

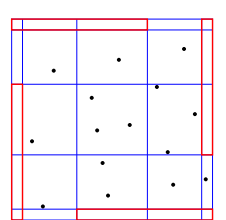

(b)

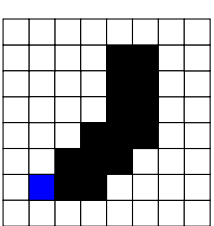

(c)
Fig. 13 (a) An initial finite set $S \subset \mathbb{Z}^{2}$, colored in black and red. (b) The remainder map image of $S$, i.e. $\rho(\mathbf{p})$ for all $\mathbf{p} \in S$, under $U$-given by parameters $\left(\frac{1}{4}, 0, \arccos \frac{35}{37}\right)$. Since no point $\rho(\mathbf{p})$ lies within the non-injective zone $\mathcal{F}$, we have a visual proof that $U$ restricted to $S$ is injective. (c) The set $S$ under the digitized rigid motion colored in black and blue. In (a) (resp. (b)) the point in the middle of the red (resp. blue) square has coordinates $(3,5)$ (resp. $(1,6))$.

\subsection{The backward algorithm}

In this section, we consider a square finite set $S$ as the input; this setting is not abnormal, as we can find a square bounding box for any finite set. The strategy of the proposed backward algorithm consists of: Step 1: for a given $U$, i.e. a Pythagorean triple and a rational translation vector, enumerate all the points in the non-injective zones $W=\{\mathbf{w} \mid$ $\mathbf{w} \in \mathcal{G}^{\prime} \cap \mathcal{F}$; Step 2: compute all their preimages, i.e., $W^{-1}=\left\{\rho^{-1}(\mathbf{w}) \mid \mathbf{w} \in W\right\}$; Step 3: determine among them those in $S$, i.e., $S \cap W^{-1}$.

Step 1

As explained in Section 4, the cyclic group $\mathcal{L}$ is generated by $\boldsymbol{\psi}=\left(\frac{p}{c}, \frac{q}{c}\right)$ and $\boldsymbol{\omega}=\left(-\frac{q}{c}, \frac{p}{c}\right)$, and $\mathcal{L}^{\prime}$ is its translation (modulo $\mathbb{Z}^{2}$ ). Therefore, all the points in $\mathcal{L}^{\prime}$ can be expressed as $\mathbb{Z} \boldsymbol{\psi}+\mathbb{Z} \boldsymbol{\omega}+\{\mathbf{t}\}$. To find these points of $\mathcal{L}^{\prime}$ in the non-injective zones, let us focus on $f_{\downarrow}^{2}$, given in Lemma 2. (Note that a similar discussion is valid for any other non-injective zones given by Lemma 2). The set of remainder points $\mathbb{Z} \boldsymbol{\psi}+\mathbb{Z} \boldsymbol{\omega}+\{\mathbf{t}\}$ lying in $f_{\downarrow}^{2}$ is then formulated by the following four linear inequalities — critical lines bounding $f_{\downarrow}^{2}$ —and we define the non-injective remainder index set $C_{\downarrow}$ such that

$C_{\downarrow}=\left\{\begin{array}{l|l}(i, j) \in \mathbb{Z}^{2} & \begin{array}{l}-\frac{1}{2}<\frac{p}{c} i-\frac{q}{c} j+\left\{t_{x}\right\}<\frac{1}{2}-\frac{2 p q}{p^{2}+q^{2}}, \\ \frac{p^{2}-q^{2}}{p^{2}+q^{2}}-\frac{1}{2}<\frac{q}{c} i+\frac{p}{c} j+\left\{t_{y}\right\}<\frac{1}{2}\end{array}\end{array}\right\}$.

Solving the system of inequalities in Equation (8) consists of finding all pairs $(i, j) \in \mathbb{Z}^{2}$ inside the given rectangle. This is carried out by mapping 
$\mathbb{Z} \boldsymbol{\psi}+\mathbb{Z} \boldsymbol{\omega}+\{\mathbf{t}\}$ to $\mathbb{Z}^{2}$ using a similarity, denoting by $\hat{f}_{\downarrow}^{2}$ the image of $f_{\downarrow}^{2}$ under this transformation (Figure 14).

To determine all the integer points in $(i, j) \in C_{\downarrow}$, we first consider the upper and lower corners of the rectangular region $\hat{f}_{\downarrow}^{2}$ given by Equation (8), i.e. $\left(\frac{p-3 q}{2}, \frac{p-q}{2}\right)$ and $\left(\frac{q-p}{2}, \frac{p+q}{2}\right)$. Then, we find all the horizontal lines $j=k$ where $k \in \mathbb{Z} \cap\left(\frac{p-q}{2}, \frac{p+q}{2}\right)$. For each line $j=k$, we obtain the two intersections with the boundary of $\hat{f}_{\downarrow}^{2}$ as the maximal and minimal integers for $i$ (see Figure 14(a)).

The complexity of this step depends on the number of integer lines crossing $\hat{f}_{\downarrow}^{2}$, which is $q$, and thus it is $\mathcal{O}(q)$.

\section{Step 2}

We seek the set of all preimages of $i \boldsymbol{\psi}+j \boldsymbol{\omega}+\{\mathbf{t}\}$ for each $(i, j) \in C_{\downarrow}$, or equivalently, preimages of $i \boldsymbol{\psi}+j \boldsymbol{\omega}$ by the translationless remainder map (The fact that this point is in $f_{\downarrow}^{2}$ plays no role in this step.). This is a Diophantine system (modulo $\mathbb{Z}^{2}$ ), and the set of preimages of a point $i \boldsymbol{\psi}+j \boldsymbol{\omega}+\{\mathbf{t}\}$ is given by a sublattice of $\mathbb{Z}^{2}$ :

$\mathbb{T}(i, j)=p \frac{\mu-v}{2}\left(\begin{array}{l}i \\ j\end{array}\right)+\mathbb{Z}\left(\begin{array}{c}a \\ -b\end{array}\right)+\mathbb{Z}\left(\begin{array}{c}c \sigma \\ c \tau\end{array}\right)$

where $\mu, v$ and $\sigma, \tau$ are the Bézout coefficients satisfying $\mu p^{2}+v q^{2}=1$, and $\sigma a+\tau b=1$, respectively.

To find these Bézout coefficients, we use the extended Euclidean algorithm. The time complexity of finding $\mu$ and $v$ (resp. $\sigma$ and $\tau$ ) is $\mathcal{O}(\log q)$ (resp. $\mathcal{O}(\log \min (a, b))[4]$. As the Bézout coefficients are computed once for all $(i, j) \in C_{\downarrow}$, the time complexity of Step 2 is $\mathcal{O}(\log q)+\mathcal{O}(\log \min (a, b))=$ $\mathcal{O}(\log \min (a, b))$.

\section{Step 3}

We now consider the union of lattices $\mathbb{T}(i, j)$ for all couples $(i, j)$ in $C_{\downarrow}$ obtained in Step 1 . To find their intersection with $S$, we apply to each an algorithm similar to Step 1 - with an affine transformation mapping the basis $\left(\begin{array}{c}a \\ -b\end{array}\right),\left(\begin{array}{c}c \sigma \\ c \tau\end{array}\right)$ to $\left(\begin{array}{l}1 \\ 0\end{array}\right),\left(\begin{array}{l}0 \\ 1\end{array}\right)$ and $p \frac{\mu-v}{2}\left(\begin{array}{l}i \\ j\end{array}\right)$ to $\left(\begin{array}{l}0 \\ 0\end{array}\right)$. Thus, a square $S$ maps to a quadrangular $\hat{S}$ after such an affine transformation, and we find the set of integer points in $\hat{S}$. Note that

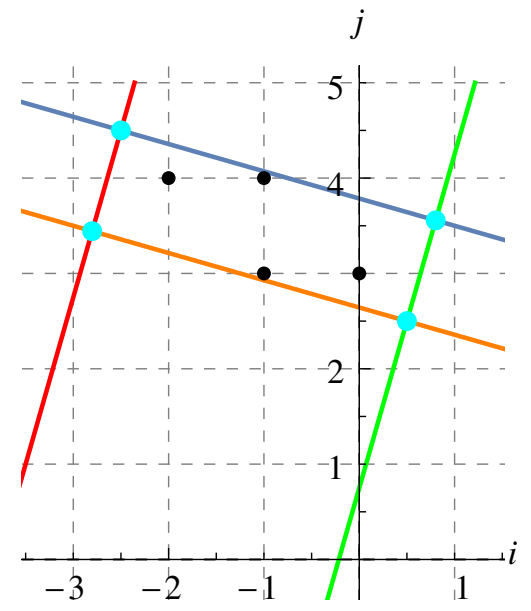

(a)

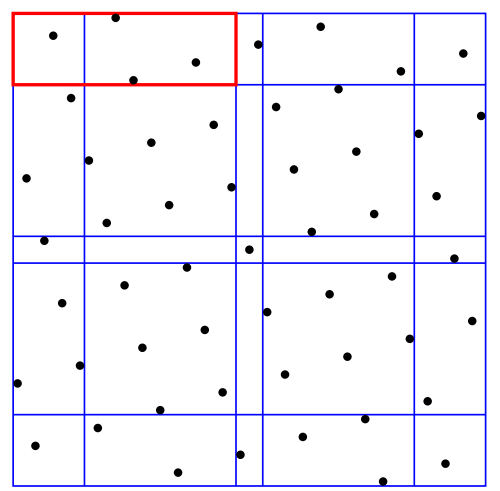

(b)

Fig. 14 (a) Geometrical interpretation of the system of linear inequalities in Equation (8), in the $(i, j)$-plane for $(p, q)=(7,2)$. The region surrounded by the four lines is $\hat{f}_{\downarrow}^{2}$, and the integer points within are marked by black circles. (b) The remainder range, $\mathcal{G}^{\prime}$ and $f_{\downarrow}^{2}$ illustrated by a red square, which corresponds to $\hat{f}_{\downarrow}^{2}$ in (a).

the involved transformation is the same for all the lattices, up to a translation.

The complexity of listing all the preimages is given by $\left|C_{\downarrow}\right|$ times the number of horizontal lines $j=k, k \in \mathbb{Z}$, intersecting $\hat{S}$, denoted by $K$. The cardinality of $C_{\downarrow}$ is related to the area of $f_{\downarrow}^{2}$ given by $\frac{2 q^{2}(p-q)^{2}}{\left(p^{2}+q^{2}\right)^{2}}$, which cannot be larger than $\frac{3}{2}-\sqrt{2}$. As $\left|\mathcal{L}^{\prime}\right|=c$ and $\left|C_{\downarrow}\right|=\left|\mathcal{L}^{\prime} \cap f_{\downarrow}^{2}\right|,\left|C_{\downarrow}\right| \leq\left(\frac{3}{2}-\sqrt{2}\right) c$. On the other hand, $K$ is bounded by $d_{S} / c$, where $d_{S}$ stands for the diagonal of $S$. As the complexity of $d_{S}$ is given by $\mathcal{G}(\sqrt{|S|})$, the final complexity of Step 3 is $\mathcal{O}(\sqrt{|S|})$. 
Remark 5 A possible refinement consists of ruling out false positives at border points $\mathbf{p}$ of $S$, by checking whether $\mathbf{p}+\mathbf{d}_{*}$ belongs to $S$, where $\mathbf{d}_{*}$ is given by the above procedure (thus avoiding the case when $\mathbf{p}$ and $\mathbf{p}+\mathbf{d}_{*}$ are mapped to the same point but $\mathbf{p}+\mathbf{d}_{*}$ is not in $S$ ). This can be achieved during Step 3.

All the steps together allow us to state that the backward algorithm, whose time complexity is $\mathcal{O}(q+\log \min (a, b)+\sqrt{|S|})$, identifies non-injective points in finite square sets.

Remark 6 Even though the backward algorithm works with squares, one can approximate any set $S$ by a union of squares and run the backward algorithm on each of them. There can be false positives; however these can be discarded one by one by verifying whether they lie in $S$ or not.

The proposed algorithms differ from a simple injectivity verification, which can be implemented using a multimap as a data structure, where each key represents a point of the transformed space and each value associated with a single key represents a set of its preimages. Since the usual complexity of operations defined on a multimap is $(\Theta(\log n)-n$ standing for the number of keys - this strategy provides a linear time complexity with respect to the size of the input digital set; nevertheless, it requires more memory than the forward or backward algorithms. Note that from a practical point of view the choice between the forward and the backward algorithm depends on the size of the input digital set $S$ and the parameters of the rational rotation. Indeed, when the cardinality of $S$ is relatively low and the cardinality of $\mathcal{L}_{\mathcal{L}}$ (actually $\mathcal{L}_{\mathcal{\prime}}^{\prime} \cap \mathcal{F}$ ) is relatively high, the forward algorithm is usually a better choice than the backward algorithm and vice versa ${ }^{1}$.

\section{Finding the local bijectivity angle interval}

The algorithms discussed in the previous section can verify if a digitized rigid motion restricted to a finite digital set $S$ is bijective. Such a digitized rigid

\footnotetext{
1 Our implementation of the forward and backward algorithms can be downloaded from http://doi .org/10. 5281 /zenodo. 248742
}

motion is given by a triplet of parameters $\left(t_{1}, t_{2}, \theta\right)-$ where $\theta$ is a Pythagorean angle and $t_{1}, t_{2} \in \mathbb{Q}$. In this section, we consider the problem of finding a range of parameters such that the corresponding digitized rigid motions remain bijective when restricted to $S$. More precisely, we start with a given digitized rigid motion which is bijective when restricted to $S$, and we focus on finding neighboring values around the triplet $\left(t_{1}, t_{2}, \theta\right)$ under the condition that each digitized rigid motion from this range ensures bijectiviy on $S$.

Such a problem can be seen as an optimization problem, namely, finding a maximal ball $\mathcal{B}_{\epsilon}\left(\left(t_{1}, t_{2}, \theta\right)\right)$ of radius $\epsilon$, centered at a point $\left(t_{1}, t_{2}, \theta\right)$ and placed in the space $\left[-\frac{1}{2}, \frac{1}{2}\right)^{2} \times\left[0, \frac{\pi}{4}\right)$ with a restriction that any digitized rigid motion represented by a point of parameters $\mathbf{v} \in \mathcal{B}_{\epsilon}\left(\left(t_{1}, t_{2}, \theta\right)\right)$ remains bijective when restricted to a given finite set $S$. More formally, we look for the maximum $\epsilon$ such that for any $\mathbf{v} \in\left\{\mathbf{v} \mid\left\|\mathbf{v}-\left(t_{1}, t_{2}, \theta\right)\right\| \leq \epsilon\right\}$ the corresponding digitized rigid motion is bijective restricted to $S$.

Instead of solving this problem, that requires to consider the three parameters simultaneously, in this section, we consider a simpler, yet practically relevant, problem by separately considering the translation and rotation parts. First, we fix the translation vector $\mathbf{t}$, and find which nearby angles of rotation preserve bijectivity, by using the notion of hinge angles $[2,9,13,14]$. More precisely, we compute the largest open interval of angles which contains the initial angle $\theta$, such that for any angle $\theta^{\prime}$ in this interval, the digitized rigid motions given by $\theta^{\prime}$ and $\mathbf{t}$ remain injective on $S$. In particular, we show how such an interval can be computed from an extended version of the forward algorithm presented in Section 5. We also compute, in that interval, which angles correspond to a change in the neighborhood motion map, i.e. the local behavior of $U$ (so that $U$ changes but is still locally bijective).

Second, one can also find, for a given angle $\theta$, a range of translation vectors $\mathbf{t}$ guaranteeing that the corresponding rigid motions remain bijective. Such a strategy consists of measuring, for each point $\mathbf{p} \in S$, the distance between $\rho(\mathbf{p})$ and non-injective frames $f_{*}^{2}$, and returning the lowest distance. This problem is simple; therefore, hereafter we only con- 
sider the first problem, namely, we search for angles with a fixed translation.

\subsection{Hinge angles for rigid motions}

In his $\mathrm{PhD}$ thesis [2], Fredriksson considered digitized rotations and the transition angles which correspond to a shift in the image of an integer point $\mathbf{p} \in \mathbb{Z}^{2}$ from one digitization cell to another. These special angles were further studied - and named hinge angles - by Nouvel and Rémila [9] and by Thibault et al. [13,14]. In the sequel, we extend this notion of hinge angles to the case of rigid motions with a given translation.

Definition 3 Given a translation vector $\mathbf{t}$, an angle $\alpha$ is called a hinge angle if there exists at least one point in $\mathbb{Z}^{2}$ such that its image by a rigid motionrotation by $\alpha$ followed by the translation-has a half-integer coordinate.

A hinge angle is represented by an integer quadruple $\left(p_{1}, p_{2}, k, s\right) \in \mathbb{Z}^{3} \times\{0,1\}$, where $\left(p_{1}, p_{2}\right)$ is the original integer position of $\mathbf{p}, k+\frac{1}{2}$ stands for a half-grid line and $s$ is a binary flag which allows us to distinguish between different directions of a half-grid line, namely, $s=0$ stands for the vertical direction and $s=1$ for the horizontal one. Let $\mathcal{U}(\mathbf{p})=\left(p_{1}^{\prime}, p_{2}^{\prime}\right)$; then, in accordance with Figure 15(b), we obtain

$\cos \alpha=\frac{p_{1}\left(p_{1}^{\prime}-t_{1}\right)+p_{2}\left(p_{2}^{\prime}-t_{2}\right)}{p_{1}^{2}+p_{2}^{2}}$

and, in particular, when $\left(p_{1}^{\prime}, p_{2}^{\prime}\right)=(\lambda, k+1 / 2)$,

$\cos \alpha=\frac{p_{1} \lambda+p_{2}\left(k-t_{2}+\frac{1}{2}\right)}{p_{1}^{2}+p_{2}^{2}}$,

where $\lambda=\sqrt{p_{1}^{2}+p_{2}^{2}-\left(k-t_{2}+\frac{1}{2}\right)^{2}}$. Similarly, $\sin \alpha$ (resp. cosine/sine for $\left.\left(p_{1}^{\prime}, p_{2}^{\prime}\right)=(k+1 / 2, \lambda)\right)$ can be obtained from the sum-difference identity of trigonometric functions. Figure 15 illustrates some hinge angles of an integer point. Moreover, we consider a function $\zeta$ such that for a quadruple $\left(p_{1}, p_{2}, k, s\right)$ it returns the corresponding angle.

Lemma 3 Let $\alpha$ and $\beta$ be two hinge angles. We can check if $\alpha>\beta$ in constant time, by using only integer computations.

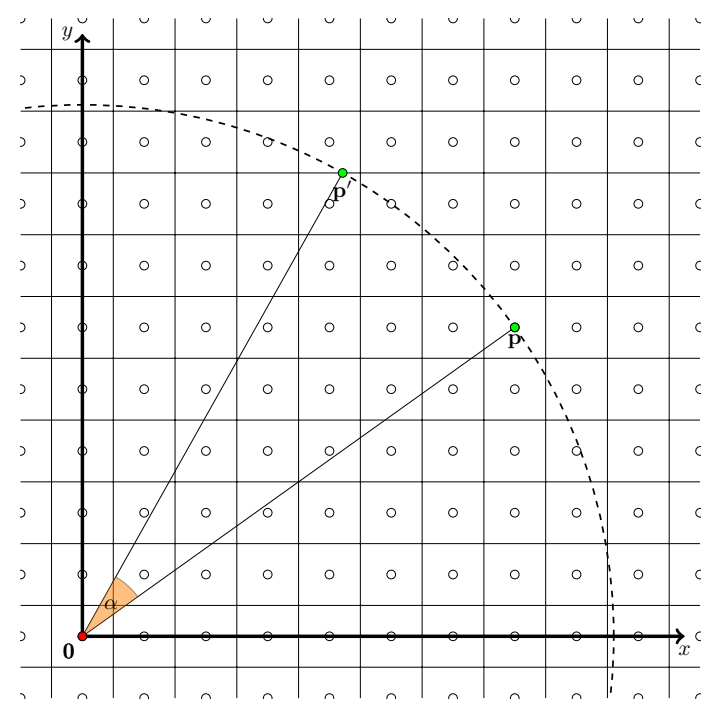

(a)

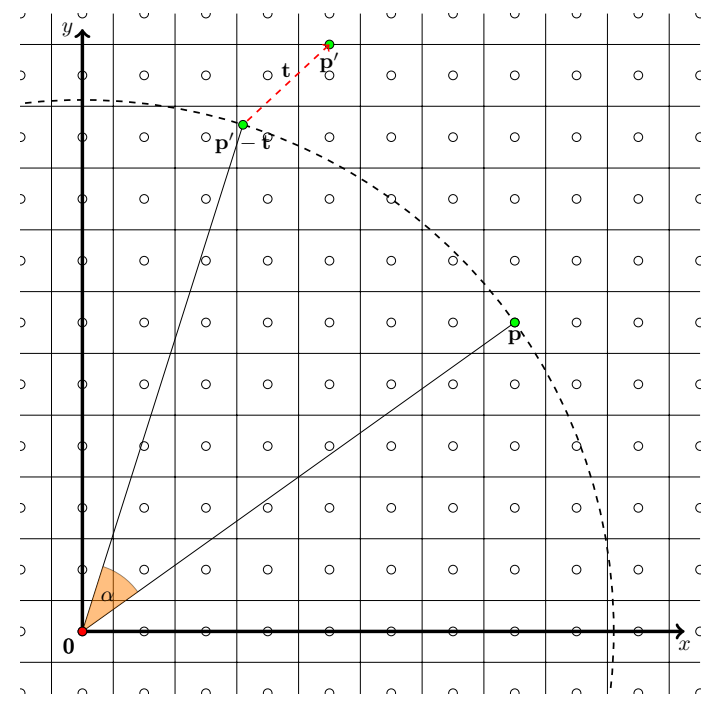

(b)

Fig. 15 Examples of rigid motions which induce transition of the point $\mathbf{p}=(7,5)$ between digitization cells; (a) $\alpha$ represented by $(7,5,7,1), \mathbf{t}=\mathbf{0}$ and (b) $\alpha$ represented by $(7,5,9,1), \mathbf{t}=\left(\frac{7}{5}, \frac{13}{10}\right)$.

Lemma 4 Let $\alpha$ be a hinge angle, and $\theta$ be a Pythagorean angle. We can check whether $\alpha>\theta$ in constant time, by using only integer computations.

Lemma 3 (resp. Lemma 4) can be proved in a similar way as [13, Theorem 3.9 and Theorem 3.8 , respectively], i.e., by considering the signs of 
the different sides of inequalities obtained from a difference of two cosines given by Equation (11) (resp. cosine give by Equation (11) and cosine of a Pythagorean angle)..

Remark 7 Nouvel and Rémila proved that for $\mathbf{t}=\mathbf{0}$, the intersection between hinge and Pythagorean angles is empty [9]. This property is generally lost for $\mathbf{t} \neq \mathbf{0}$.

Let $E_{\mathbf{t}}(\mathbf{p})=\left\{\zeta\left(p_{1}, p_{2}, k, s\right) \mid k \in \mathbb{Z}, s \in\{0,1\}\right\}$ be the ordered set of hinge angles for a given point $\mathbf{p}=\left(p_{1}, p_{2}\right) \in \mathbb{Z}^{2}$, which is different from the origin. Then, using results of Lemma 3 and 4, given a Pythagorean (or hinge) angle $\theta$, we define a function $h_{\mathbf{t}}^{<}(\mathbf{p}, \theta)\left(\right.$ resp. $\left.h_{\mathbf{t}}^{>}(\mathbf{p}, \theta)\right)$ which returns, for given $\mathbf{p} \in \mathbb{Z}^{2}$ and translation $\mathbf{t} \in \mathbb{Q}^{2}$, the closest lower (resp. upper) hinge angle to $\theta$ in $E_{\mathbf{t}}(\mathbf{p})$, namely $h_{\mathbf{t}}^{<}(\mathbf{p}, \theta)<\theta$ (resp. $\left.\theta<h_{\mathbf{t}}^{>}(\mathbf{p}, \theta)\right)$. In other words, $\nexists \alpha \in E_{\mathbf{t}}(\mathbf{p})$ such that $h_{\mathbf{t}}^{<}(\mathbf{p}, \theta)<\alpha<\theta$ (resp. $\left.\theta<\alpha<h_{\mathbf{t}}^{>}(\mathbf{p}, \theta)\right)$. Note that $h_{\mathbf{t}}^{<}(\mathbf{p}, \theta)\left(\operatorname{resp} . h_{\mathbf{t}}^{>}(\mathbf{p}, \theta)\right)$ can be determined in constant time by considering half-grid lines which bound the closest digitization cell, i.e., $C(U(\mathbf{p}))^{2}$. Figure 16 shows examples of $h_{\mathbf{t}}^{<}(\mathbf{p}, \theta)$ (resp. $\left.h_{\mathbf{t}}^{>}(\mathbf{p}, \theta)\right)$ for some $\mathbf{p} \in \mathbb{Z}^{2}$ and $\left(t_{1}, t_{2}, \theta\right)$

6.2 An algorithm for finding the local bijectivity angle interval

Let us define an ordered set $C_{\mathbf{p}} \subset E_{\mathbf{t}}(\mathbf{p})$ such that for any hinge angle $\alpha \in\left(\inf \left(C_{\mathbf{p}}\right), \sup \left(C_{\mathbf{p}}\right)\right)$, where $\sup \left(C_{\mathbf{p}}\right)$ and $\inf \left(C_{\mathbf{p}}\right)$ stand for supremum and infimum of $C_{\mathbf{p}}$, the corresponding digitized rigid motion is bijective when restricted to $\mathcal{N}_{1}(\mathbf{p})$, and $\exists i \in$ $\mathbb{N}_{+}$such that $\left(\left(\alpha_{i}<\theta<\alpha_{i+1}\right) \wedge\left(\alpha_{i}, \alpha_{i+1} \in C_{\mathbf{p}}\right)\right)$.

We shall now build iteratively, for a given Pythagorean angle $\theta$ and fixed translation vector $\mathbf{t} \in \mathbb{Q}^{2}$, an ordered set of hinge angles $C=\left(\bigcup_{\mathbf{p} \in S} E_{\mathbf{t}}(\mathbf{p})\right) \cap\left(\bigcap_{\mathbf{p} \in S}\left[\inf \left(C_{\mathbf{p}}\right), \sup \left(C_{\mathbf{p}}\right)\right]\right)$ such

\footnotetext{
2 In [14], Thibault et al. an algorithm was proposed for computing hinge angles $h_{\mathrm{t}}^{<}(\mathbf{p}, \theta)$ and $h_{\mathrm{t}}^{>}(\mathbf{p}, \theta)$-for $\mathbf{t}=\mathbf{0}$-in a logarithmic time, which can be improved to constant time while considering half-grid lines which bound the closest digitization cell, i.e., $C(U(\mathbf{p}))$. Notice that the algorithm also needs a modification in the while loop condition, such that $k_{\max }-k_{\min } \leq 1$, to avoid an infinite loop for some points, e.g., $\mathbf{p}=(1,0)$.
}

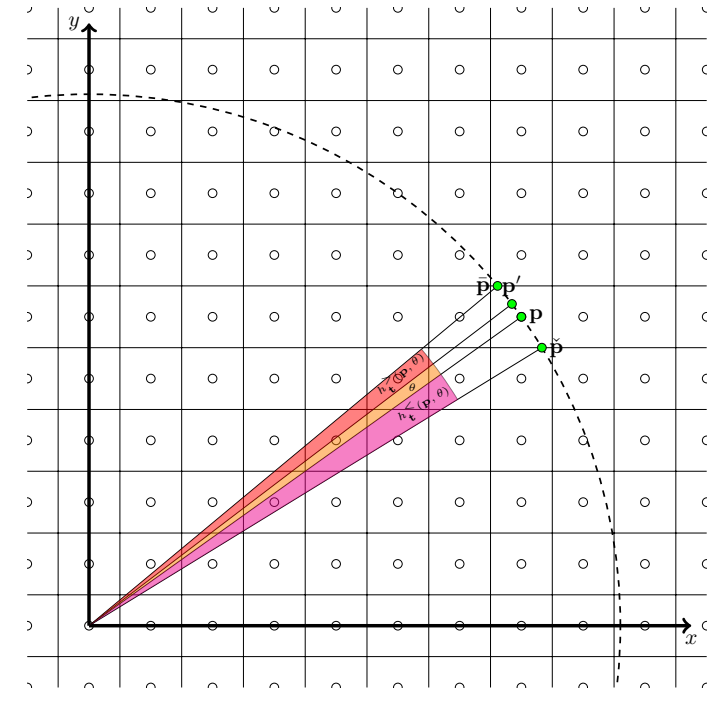

Fig. 16 Visualization of different images of the point $\mathbf{p}=$ $(7,5)$ under rigid motions given by fixed $\mathbf{t}=\mathbf{0}$ and rotations by angles: $\theta=\frac{3}{100}$ and hinge angles $h_{\mathbf{t}}^{<}(\mathbf{p}, \theta)=-\zeta(7,5,4,1)$ - clockwise rotation of $\mathbf{p}, h_{\mathbf{t}}^{>}(\mathbf{p}, \theta)=\zeta(7,5,5,1)$, represented by points $\mathbf{p}^{\prime}, \check{\mathbf{p}}$ and $\overline{\mathbf{p}}$, respectively.

that $(\inf (C), \sup (C))$ is the largest open angle interval containing $\theta$ guaranteeing that the digitized rigid motions represented by such angles and the translation vector $\mathbf{t}$ are bijective while restricted to $S$. Let $\gamma_{\mathbf{t}}^{<}=\inf (C)\left(\operatorname{resp} . \gamma_{\mathbf{t}}^{>}=\sup (C)\right)$; then we initialize the angle $\gamma_{t}^{<}\left(\operatorname{resp} . \gamma_{t}^{>}\right)$with $-2 \pi(\operatorname{resp} .2 \pi)$. To verify if the digitized rigid motion corresponding to a hinge angle $\alpha$ is non-injective, let $\operatorname{cl}\left(f_{*}^{2}\right)$ stands for the closure of a non-injective zone $f_{*}^{2}$ and let $\rho^{\alpha}(\mathbf{p})$ stands for the remainder map, where the initial angle $\theta$ has been substituted with the angle $\alpha$. Then, as $\alpha$ is a hinge angle, $\rho^{\alpha}(\mathbf{p})$ is on the border of the remainder range.

To verify if the digitized rigid motion corresponding to a hinge angle $\alpha$ is non-injective, let $c l\left(f_{*}^{2}\right)$ stand for the closure of a non-injective zone $f_{*}^{2}$. As $\alpha$ is a hinge angle, $\rho^{\alpha}(\mathbf{p})$ is on the border of the remainder range. To efficiently build the ordered set $C$, let $\rho^{\alpha}(\mathbf{p})$ stand for the remainder map, where the initial angle $\theta$ has been substituted with the angle $\alpha$.

The strategy consists of iteratively verifying if any point $\mathbf{p} \in S$ belongs to a non-injective mapping under $U$. If so, we return an empty ordered set $C$ (this is just for the sake of completeness, since we 


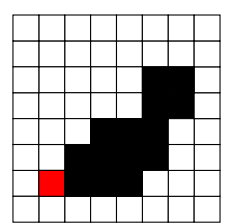

(a)

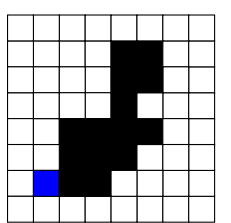

(b)

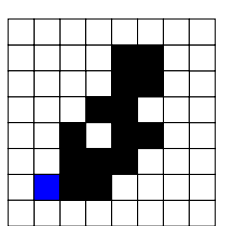

(c)
Fig. 17 (a) An initial finite set $S \subset \mathbb{Z}^{2}$, colored in black and red. (b)-(c) represent images of $S$ under digitized rigid motions for parameters obtained by the extended forward algorithm for initial parameters $\left(0,0, \arccos \frac{35}{37}\right)$, i.e., $C=$ $\{\zeta(6,7,3,0), \zeta(5,7,2,0), \zeta(8,9,4,0)\}$. (b) The image of $S$ for $\alpha \in(\zeta(6,7,3,0), \zeta(5,7,2,0))$ and (c) the image of $S$ for $\alpha \in(\zeta(5,7,2,0), \zeta(8,9,4,0))$. In (a) (resp. (b) and (c)) the point in the middle of the red (resp. blue) square has coordinates $(3,5)$ (resp. $(1,6))$.

want to apply the extended algorithm to the case when $U$ maps $S$ injectively). Otherwise, the function $h_{\mathbf{t}}^{<}(\mathbf{p}, \theta)\left(\right.$ resp. $\left.h_{\mathbf{t}}^{>}(\mathbf{p}, \theta)\right)$ is applied to update $\gamma_{\mathbf{t}}^{<}$ (resp. $\gamma_{t}^{>}$) by finding a hinge angle $\alpha \in\left[\gamma_{t}^{<}, \gamma_{t}^{>}\right]$ such that non-injectivity occurs for $\mathbf{p} \in S$. Note that at least one coordinate of $\rho^{\alpha}(\mathbf{p})$ is on the border of the remainder range. Moreover, intermediate hinge angles which do not induce non-injectivity are stored in $C$ : these angles induce different images of $S$ under digitized rigid motions.

Figure 17 presents different images of some finite set $S$ under digitized rigid motions represented by parameters obtained from the extended forward algorithm. Note that for the example represented by Figure 13, i.e., the finite set $S$ (see Figure 13(a)) and initial parameters $\left(\frac{1}{4}, 0, \arccos \frac{35}{37}\right)$, where $\theta=\arccos \frac{35}{37}$ is given by the Pythagorean triple $(35,12,27)$, the extended forward algorithm gives $C=\{(3,5,1,0),(7,7,4,0)\}$. This process is summarized in the extended forward algorithm, below. The time complexity of this algorithm is given by the number of hinge angles for the furthest point from the origin $\mathbf{p}=\left(p_{1}, p_{2}\right) \in S$ which, for a given $\mathbf{t}$, is lower than $n=\left\lfloor\sqrt{p_{1}^{2}+p_{2}^{2}}+\frac{1}{2}\right\rfloor[13$, Chapter $3]$ and the cardinality of a finite set $S$, leading to $\mathcal{O}(n|S|)$, which is rewritten by $\mathcal{O}(\sqrt{|S|}|S|)$ if we assume that $S$ forms a square.

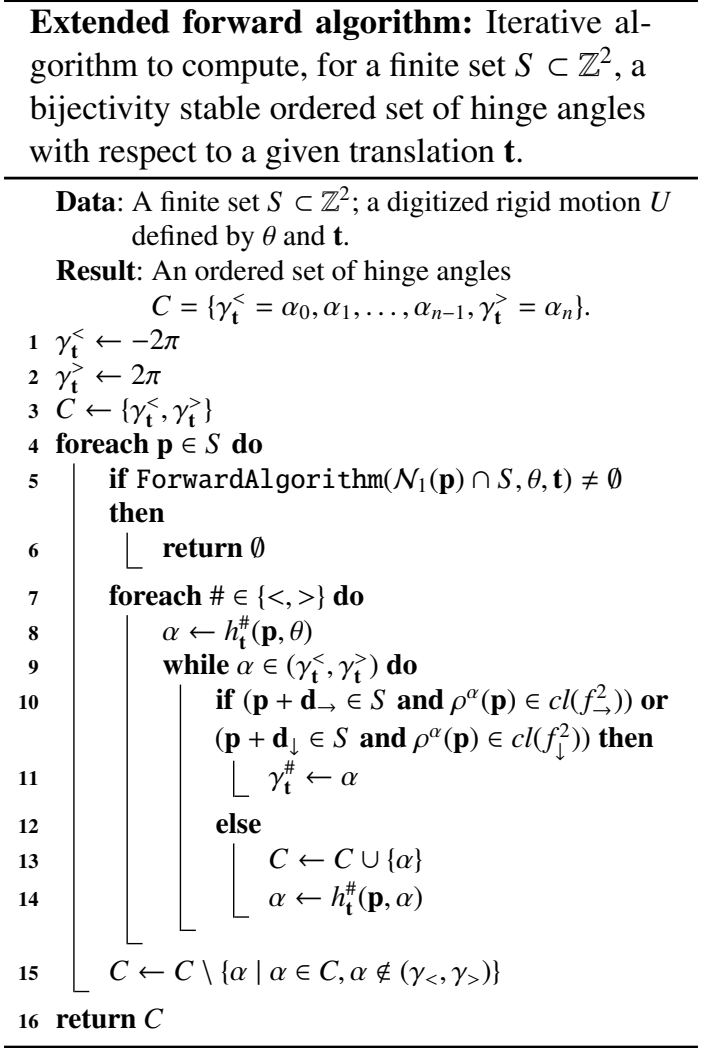

\section{Conclusion}

In this article, we have extended the neighborhood motion maps to rigid motions and any neighborhood-previously proposed by Nouvel and Rémila [6] for digitized rotations and 4neighborhood-and we have shown that these notations are useful to characterize the bijectivity of rigid motions on $\mathbb{Z}^{2}$.

We first proved some necessary and sufficient conditions of bijective rigid motions on $\mathbb{Z}^{2}$, i.e., rigid motions such that no point $\mathbf{p} \in \mathbb{Z}^{2}$ has its image $\rho(\mathbf{p})$ in either non-injective or non-surjective zones. Then, from a more practical point of view, we focused on finite sets of $\mathbb{Z}^{2}$ rather than the whole $\mathbb{Z}^{2}$. In particular, we proposed two efficient algorithms for verifying whether a given digitized rigid motion is bijective when restricted to a finite set $S$. On the one hand, the forward algorithm consists of checking if points of $S$ have preimages in non-injective zones. On the other hand, we used a 
reverse strategy to propose the backward algorithm consisting of the identification of points in $\mathcal{L}^{\prime} \cap \mathcal{F}$ and their preimages in $S$. The complexities of the forward and backward algorithms are $\mathcal{\Theta}(|S|)$ and $\mathcal{O}(q+\log \min (a, b)+\sqrt{|S|})$, respectively. We also showed that such a verification could be extended to the problem of finding a range of parameters which preserve bijectivity of digitized rigid motions restricted to a finite set. The proposed algorithm is based on an extension of hinge angles $[9,14]$ to rigid motions. This algorithm has time complexity $\mathcal{C}(\sqrt{|S|}|S|)$.

Our main perspective is to extend the proposed framework to $3 \mathrm{D}$ digitized rigid motions. The main difficulty, with respect to the study of 2D digitized rigid motions, lies in the lack of a natural order of critical planes and high dimensionality of the parameter space. We recently proposed preliminary results on these topics in [10].

\section{References}

1. Anglin, W.S.: Using Pythagorean triangles to approximate angles. American Mathematical Monthly 95(6), 540-541 (1988)

2. Fredriksson, K.: Rotation Invariant Template Matching. Ph.D. thesis, University of Helsinki (2001)

3. Galarza, A.I.R., Seade, J.: Introduction to Classical Geometries. Birkhäuser (2007)

4. Hunter, D. J.: Essentials of Discrete Mathematics, Second Edition. Jones \& Bartlett Learning (2010)

5. Jacob, M.A., Andres, E.: On discrete rotations. In: 5th Int. Workshop on Discrete Geometry for Computer Imagery, pp. 161-174 (1995)

6. Nouvel, B., Rémila, E.: On colorations induced by discrete rotations. In: DGCI, Proceedings, Lecture Notes in Computer Science, vol. 2886, pp. 174-183. Springer (2003)

7. Nouvel, B., Rémila, E.: Characterization of bijective discretized rotations. In: IWCIA, Proceedings, Lecture Notes in Computer Science, vol. 3322, pp. 248-259. Springer (2004)

8. Nouvel, B., Rémila, E.: Configurations induced by discrete rotations: Periodicity and quasi-periodicity properties. Discrete Applied Mathematics 147(2-3), 325-343 (2005)

9. Nouvel, B., Rémila, E.: Incremental and transitive discrete rotations. In: IWCIA, Lecture Notes in Computer Science, vol. 4040, pp. 199-213. Springer (2006)

10. Pluta, K., Moroz, G., Kenmochi, Y., Romon, P.: Quadric arrangement in classifying rigid motions of a 3D digital image. In: CASC, Lecture Notes in Computer Science, vol. 9890, pp. 426-443 (2016)
11. Pluta, K., Romon, P., Kenmochi, Y., Passat, N.: Bijective rigid motions of the 2D Cartesian grid. In: DGCI, Lecture Notes in Computer Science, vol. 9647, pp. 359371. Springer (2016)

12. Roussillon, T., Cœurjolly, D.: Characterization of bijective discretized rotations by Gaussian integers. Research report, LIRIS UMR CNRS 5205 (2016). URL https://hal .archives-ouvertes . fr/hal-01259826

13. Thibault, Y.: Rotations in $2 \mathrm{D}$ and $3 \mathrm{D}$ discrete spaces. Ph.D. thesis, Université Paris-Est (2010)

14. Thibault, Y., Kenmochi, Y., Sugimoto, A.: Computing upper and lower bounds of rotation angles from digital images. Pattern Recognition 42(8), 1708-1717 (2009)

15. Yilmaz, A., Javed, O., Shah, M.: Object tracking: A survey. ACM Computing Surveys 38(4) (2006) 


\section{A Neighborhood motion maps for $\mathcal{G}_{1}^{U}$ (4-neighborhood case)}

Neighborhood motion maps for $\mathcal{G}_{1}^{U}$ are depicted as label maps, for $\theta \in\left(0, \frac{\pi}{6}\right)$ in Figure 18; and $\theta \in\left(\frac{\pi}{6}, \frac{\pi}{4}\right)$ in Figure 19. Some neighborhood motion maps are symmetric with respect to the origin, i.e., neighborhood motion map of the indices $(0,0)$.

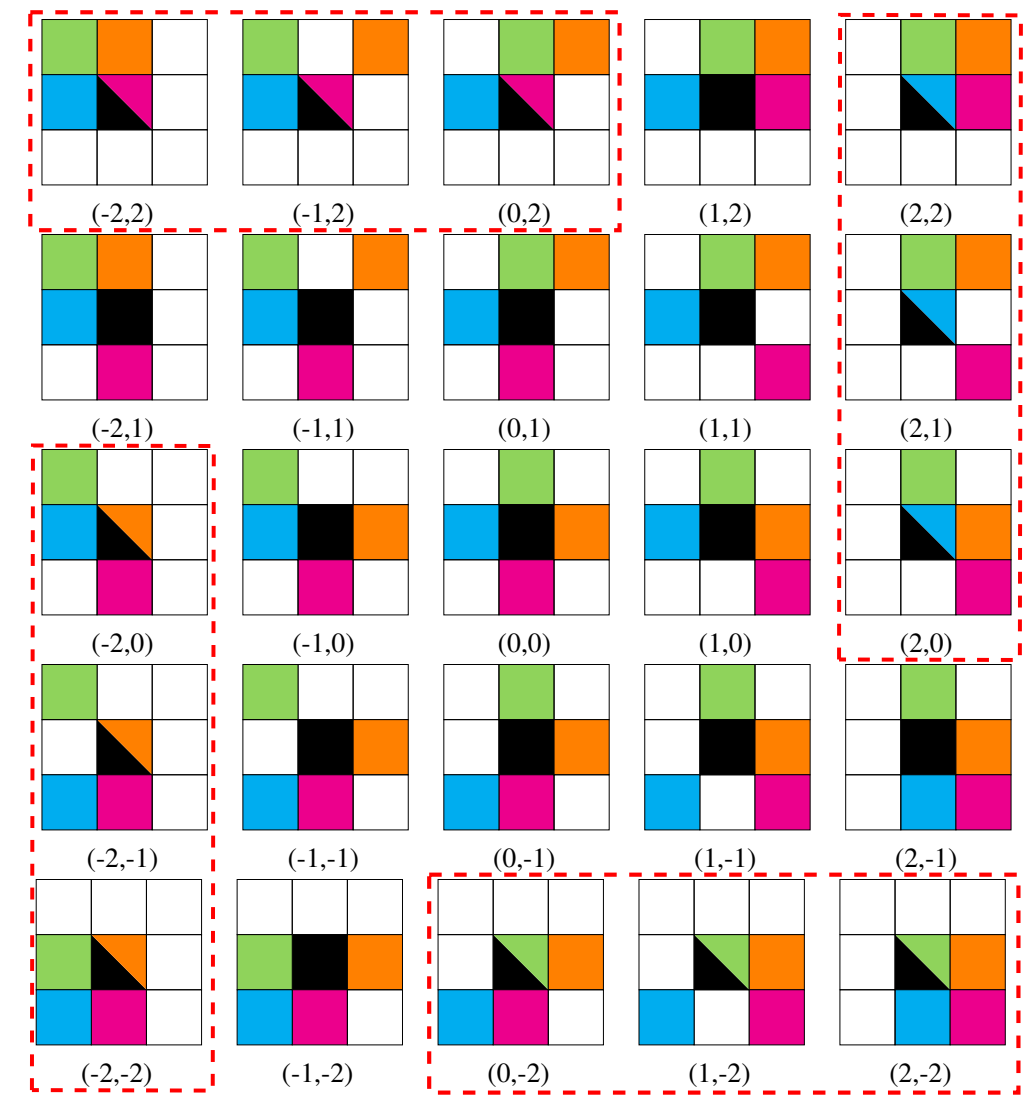

Fig. 18 Neighborhood motion maps of $\mathcal{G}_{1}^{U}$, as label maps, for $\theta \in\left(0, \frac{\pi}{6}\right)$. Each label $(i, j)$ corresponds to the frame $f_{i, j}^{\theta}$. Neighborhood motion maps which correspond to non-injective zones are marked by red, dashed, frames. 


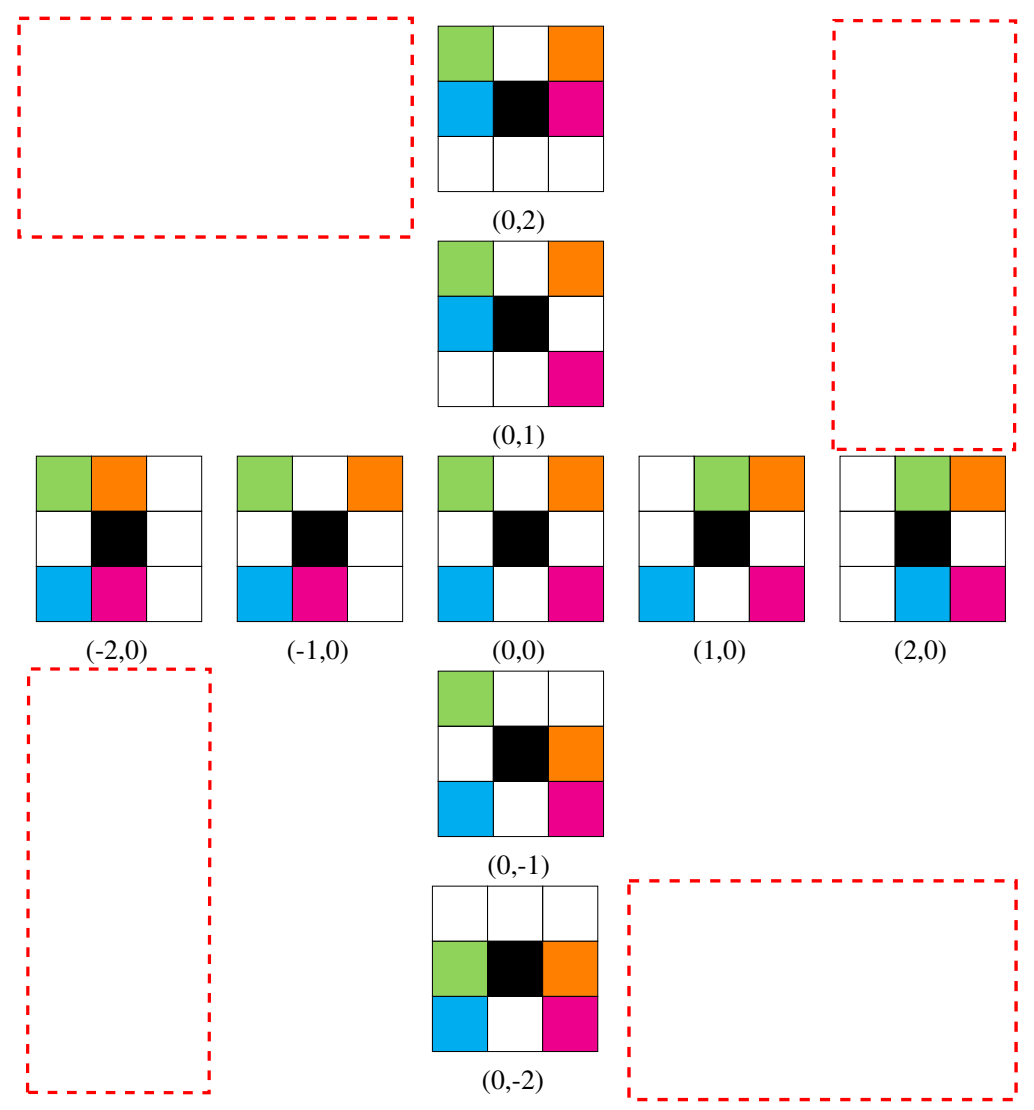

Fig. 19 Neighborhood motion maps of $\mathcal{G}_{1}^{U}$, as label maps, for $\theta \in\left(\frac{\pi}{6}, \frac{\pi}{4}\right)$ that differ from these for $\theta \in\left(0, \frac{\pi}{6}\right)$. Each label $(i, j)$ corresponds to the frame $f_{i, j}^{\theta}$. Neighborhood motion maps which correspond to non-injective zones are marked by red, dashed, frames. 


\section{B Neighborhood motion maps for $\mathcal{G}_{2}^{U}$ (8-neighborhood case)}

Neighborhood motion maps for $\mathcal{G}_{2}^{U}$ are depicted as label maps, for $\theta \in\left(0, \alpha_{1}\right)$ in Figure 20; $\theta \in\left(\alpha_{1}, \alpha_{2}\right)$ in Figure 21; $\theta \in\left(\alpha_{2}, \alpha_{3}\right)$ in Figure 22; $\theta \in\left(\alpha_{3}, \alpha_{4}\right)$ in Figure 23; and $\theta \in\left(\alpha_{4}, \frac{\pi}{4}\right)$ in Figure 24. For more information about angles $\alpha_{n}, n \in[1,4]$ we refer the reader to Figure 10. Some neighborhood motion maps are symmetric with respect to the origin, i.e., the neighborhood motion map of the indices $(0,0)$.

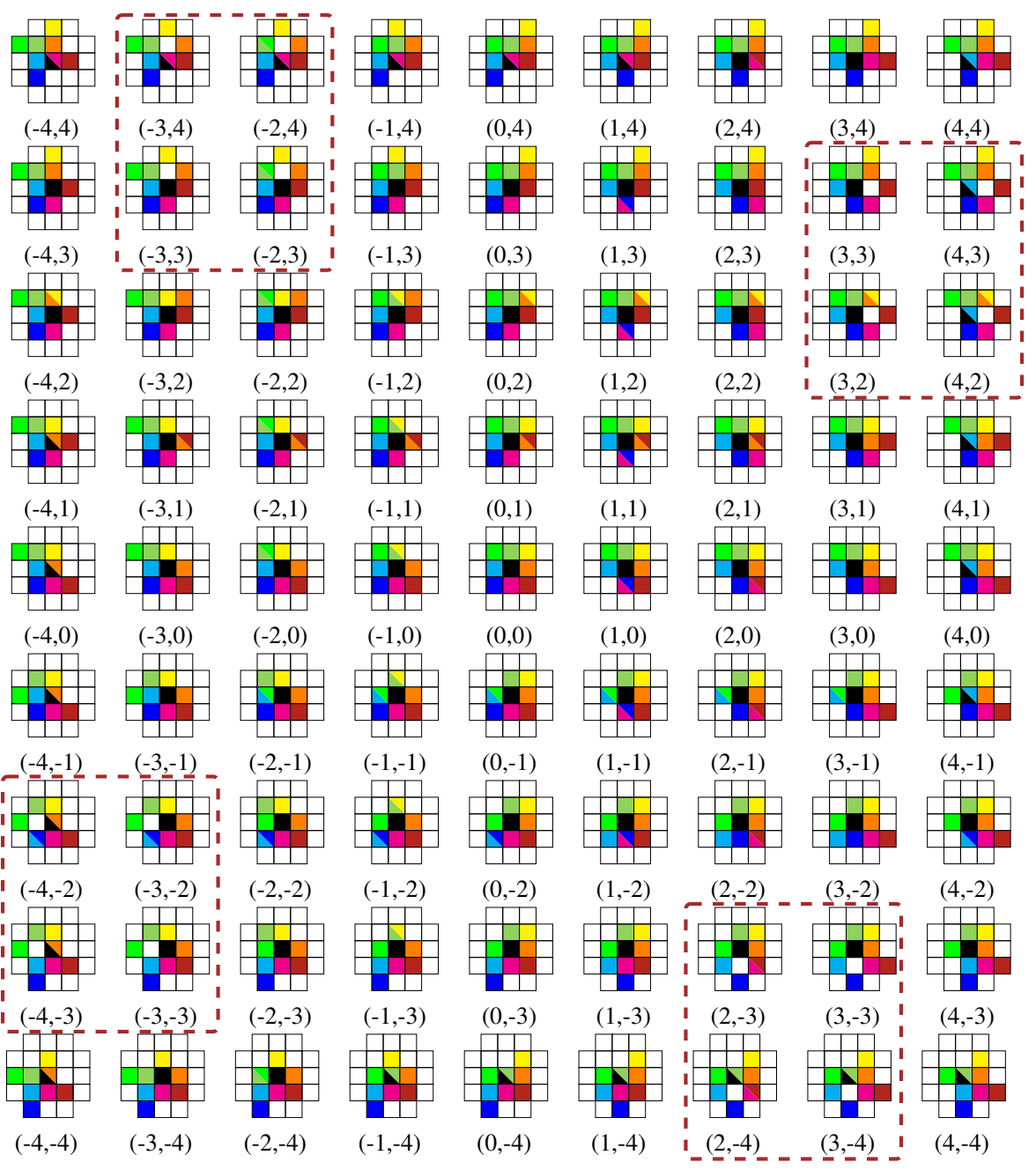

Fig. 20 Neighborhood motion maps of $\mathcal{G}_{2}^{U}$, as label maps, for $\theta \in\left(0, \alpha_{1}\right)$. Each label $(i, j)$ corresponds to the frame $f_{i, j}^{\theta}$. Neighborhood motion maps which correspond to non-injective zones are marked by brown, dashed, frames. 

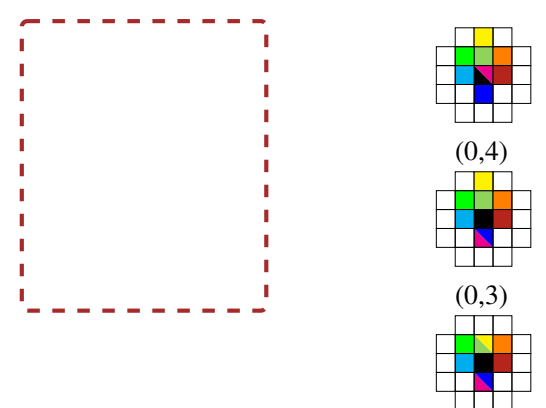

$(0,2)$
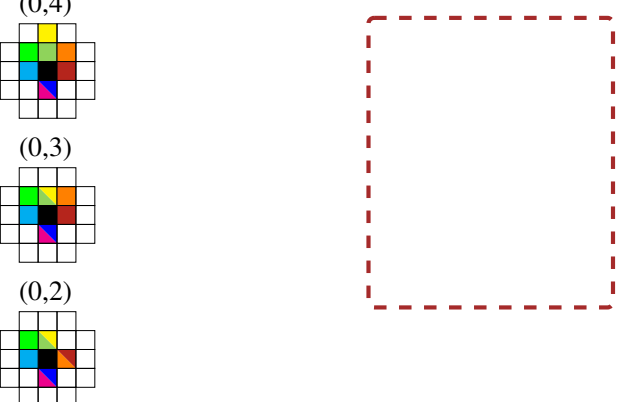

$(0,1)$
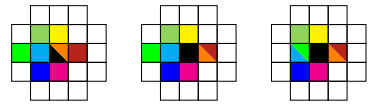

$(-3,0)$

$(-2,0)$
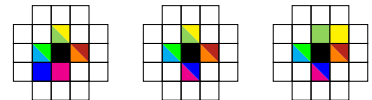

$(0,0)$

$(1,0)$
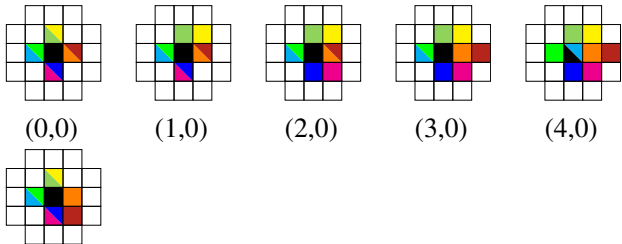

$(2,0)$

$(3,0)$

$(4,0)$

$(0,-1)$

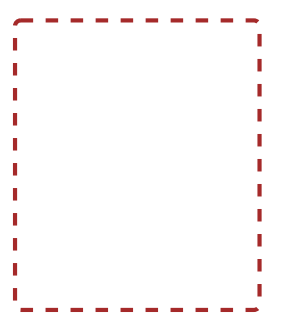

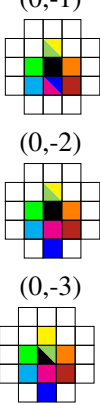

$(0,-4)$

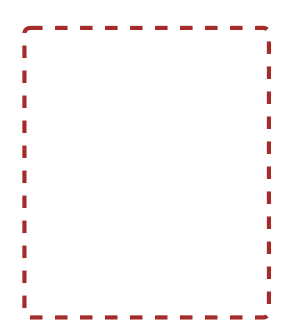

Fig. 21 Neighborhood motion maps $\mathcal{G}_{2}^{U}$, as label maps, for $\theta \in\left(\alpha_{1}, \alpha_{2}\right)$ that differ from those for $\theta \in\left(0, \alpha_{1}\right)$. Each label $(i, j)$ corresponds to the frame $f_{i, j}^{\theta}$. Neighborhood motion maps which correspond to non-injective zones are marked by brown, dashed, frames. 


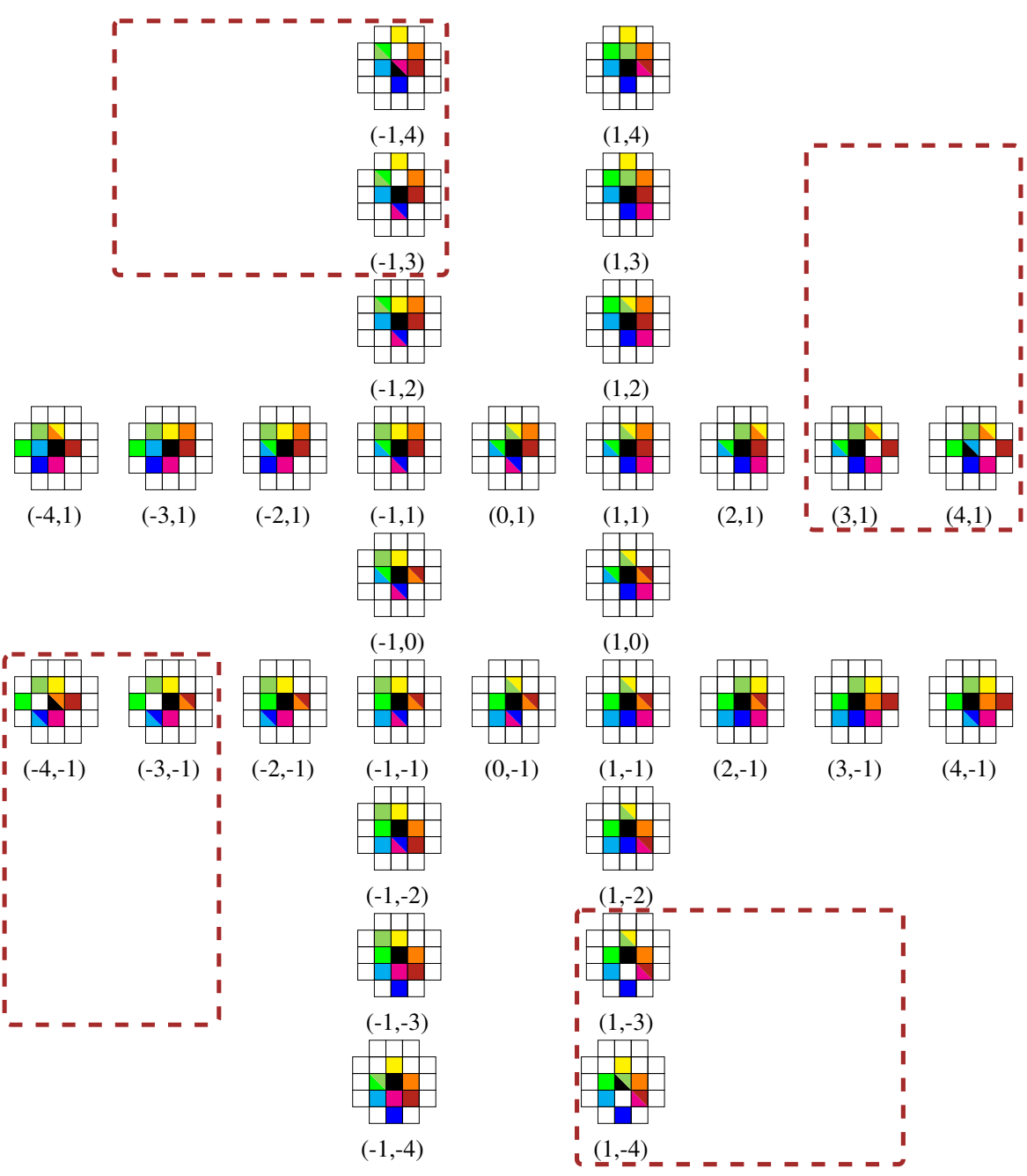

Fig. 22 Neighborhood motion maps $\mathcal{G}_{2}^{U}$, as label maps, for $\theta \in\left(\alpha_{2}, \alpha_{3}\right)$ that differ from those for $\theta \in\left(\alpha_{1}, \alpha_{2}\right)$. Each label $(i, j)$ corresponds to the frame $f_{i, j}^{\theta}$. Neighborhood motion maps which correspond to non-injective zones are marked by brown, dashed, frames. 


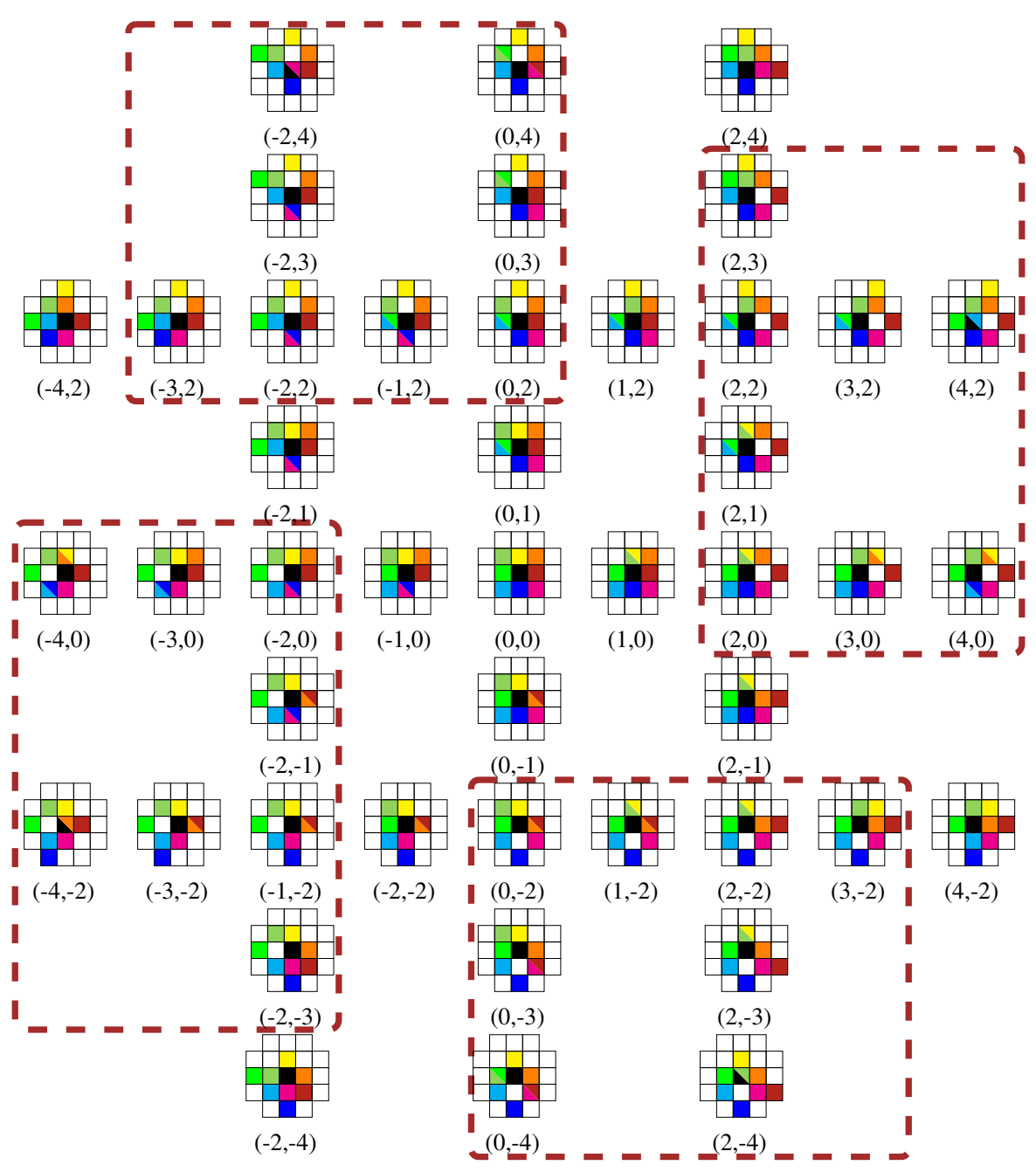

Fig. 23 Neighborhood motion maps $\mathcal{G}_{2}^{U}$, as label maps, for $\theta \in\left(\alpha_{3}, \alpha_{4}\right)$ that differ from those for $\theta \in\left(\alpha_{2}, \alpha_{3}\right)$. Each label $(i, j)$ corresponds to the frame $f_{i, j}^{\theta}$. Neighborhood motion maps which correspond to non-injective zones are marked by brown, dashed, frames. 


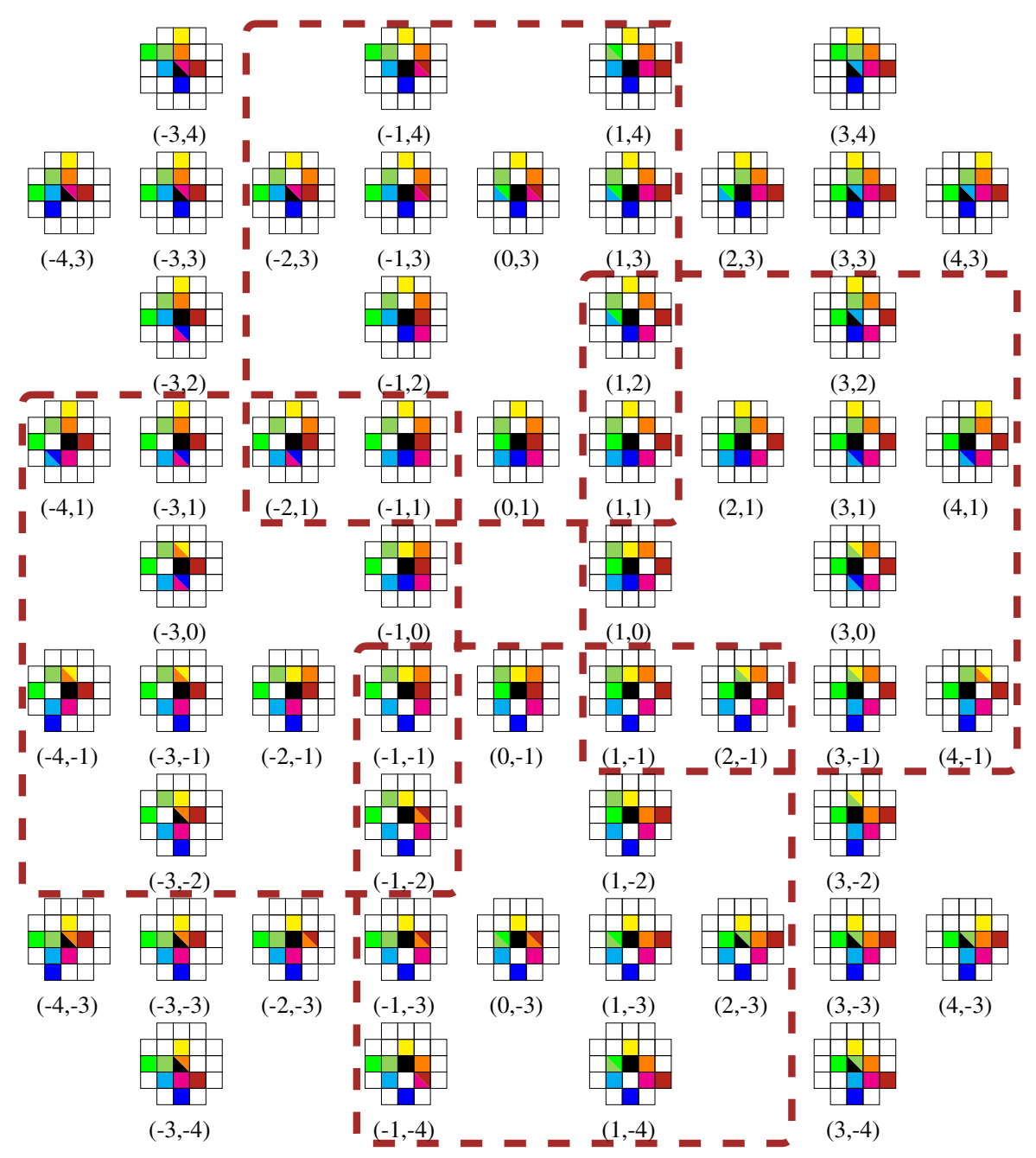

Fig. 24 Neighborhood motion maps $\mathcal{G}_{2}^{U}$, as label maps, for $\theta \in\left(\alpha_{4}, \frac{\pi}{4}\right)$ that differ from those for $\theta \in\left(\alpha_{3}, \alpha_{4}\right)$. Each label $(i, j)$ corresponds to the frame $f_{i, j}^{\theta}$. Neighborhood motion maps which correspond to non-injective zones are marked by brown, dashed, frames. 